\title{
Fuzzy Formation Control and Collision Avoidance for Multiagent Systems
}

\author{
Yeong-Hwa Chang, ${ }^{1}$ Chun-Lin Chen, ${ }^{1}$ Wei-Shou Chan, \\ Hung-Wei Lin, ${ }^{2}$ and Chia-Wen Chang $^{3}$ \\ ${ }^{1}$ Department of Electrical Engineering, Chang Gung University, Kwei-Shan, Tao-Yuan 333, Taiwan \\ ${ }^{2}$ Department of Electrical Engineering, Lee-Ming Institute of Technology, Taishan, Taipei 243, Taiwan \\ ${ }^{3}$ Department of Information and Telecommunication Engineering, Ming Chuan University, Kwei-Shan, Tao-Yuan 333, Taiwan \\ Correspondence should be addressed to Yeong-Hwa Chang; yhchang@mail.cgu.edu.tw
}

Received 2 December 2012; Accepted 12 January 2013

Academic Editor: Peng Shi

Copyright (C) 2013 Yeong-Hwa Chang et al. This is an open access article distributed under the Creative Commons Attribution License, which permits unrestricted use, distribution, and reproduction in any medium, provided the original work is properly cited.

This paper aims to investigate the formation control of leader-follower multiagent systems, where the problem of collision avoidance is considered. Based on the graph-theoretic concepts and locally distributed information, a neural fuzzy formation controller is designed with the capability of online learning. The learning rules of controller parameters can be derived from the gradient descent method. To avoid collisions between neighboring agents, a fuzzy separation controller is proposed such that the local minimum problem can be solved. In order to highlight the advantages of this fuzzy logic based collision-free formation control, both of the static and dynamic leaders are discussed for performance comparisons. Simulation results indicate that the proposed fuzzy formation and separation control can provide better formation responses compared to conventional consensus formation and potential-based collision-avoidance algorithms.

\section{Introduction}

Recently, distributed multiagent coordination has attracted much attention in many fields, where only the information available locally from its neighbors is required for each agent [1-6]. There are many applications of multiagent systems, such as autonomous unmanned aerial vehicles [7], autonomous formation flight [8], congestion-controlled communication network [9], wireless sensor network [10], and autonomous multivehicle formations [11]. Graph theory has been used to characterize network topologies for consensus studies. A general consensus problem solving is to find a distributed control strategy such that the states of agents converge to a common value. Average-consensus problem was investigated for distributed networks with fixed and switching topologies [12]. Relying on graph theory, matrix theory, and control theory, the analysis of consensus protocols was thoroughly discussed. In [13], an impulsive control protocol was presented for multiagent linear dynamic systems with fixed topology based on the local information of agents. A fuzzy sliding-mode controller was proposed to investigate the formation control problem in directed graphs [14]. Cai et al. [15] addressed the controllability improvement problem for two types of linear time-invariant dynamic multiagent systems by adjusting the configuration of graphs. A general case of leader-following consensus problems under fixed and switching topologies was discussed in [16]. The second-order agents under switching topology were concerned in [17], where the condition of communication delays was determined for achieving consensus. The consensus problem for a group of high-order dynamic agents with switching topology and time-varying communication delays was discussed in [18]. In the work of [19], linear consensus protocol and saturated consensus protocol were presented for the consensus problem of heterogeneous multiagent system. The heterogeneous multiagent system consists of first-order and secondorder integrator agents.

Lately, the fuzzy logic control which consists of linguistic control rules is a technique to design controllers based on human expert knowledge and experience. This technique 
is a good alternative to overcome the difficulties in the requirement of exact mathematics models for plants with unexpected complex dynamics and external disturbances. Although the method has been practically successful, it has proved extremely difficult to develop a general analysis and design theory for conventional fuzzy control systems. Recently, based on Takagi-Sugeno (T-S) fuzzy techniques, there have appeared in the literature a great number of results concerning stability analysis and design. T-S fuzzy techniques have been applied to many applications of interest [2025]. However, the membership functions of the above method are through manual adjustment. Lately, neural fuzzy control, combining with the capability of fuzzy reasoning to handle uncertain information and the capability of artificial neural networks to learn from processes, has been popularly addressed. In [26], a robust fuzzy neural network control (FNN) scheme including a parameter tuning algorithm was designed for a linear Maglev rail system to achieve the objective of model-free control. A neural network-based selflearning control strategy including an FNN controller and a recurrent neural network identifier was proposed for electronic throttle valves [27]. Lin and Shen [28] proposed an adaptive fuzzy neural network control scheme for a fieldoriented control permanent magnet linear synchronous motor servo-drive system to track periodic reference trajectories. In [29], an adaptive network based fuzzy inference system was presented for speed and position estimations of a permanent-magnet synchronous generator. In [30], an adaptive neurofuzzy controller was presented for the tracking control of dynamic systems with unknown nonlinearities. A recurrent fuzzy neural controller for the robust tracking of a robot manipulator with adaptive observers was addressed in [31]. A robust self-organizing neural fuzzy control scheme was proposed for a class of uncertain nonlinear MIMO systems [32]. In the study of [33], an adaptive neurofuzzy inference system was employed to identify hand motion commands based on surface electromyogram signals. In [34], a new approach was proposed for machine health condition prognosis with the integration of neurofuzzy system and Bayesian algorithms. Moreover, a T-S fuzzy-neural model was adopted for identification and robust adaptive control of an antilock braking system [35]. In addition, a hybrid evolutionary algorithm using fuzzy rules to adjust optimization parameters was proposed in [36].

In multiagent networks, the problem of collision avoidance is also an important and interesting topic that is worthy of being discussed. A cooperative control law was proposed for general nonlinear dynamic models to guarantee collisionfree conflict resolution [37]. In [38], a fuzzy logic was designed for potential functions to achieve the separation control with input constrains. In the work of [39], a flocking algorithm was presented for separation forces generated to avoid collisions with external obstacles. A modified avoidance function was proposed for nonlinear Lagrange systems to achieve collision avoidance with bounded disturbances [40]. In [41], a potential field method was discussed for mobile robots to solve the local minimum problem. In addition, Wang and $\mathrm{Gu}$ [42] presented a fuzzy potential force for the separating potential function in flocking control.
However, only few of existing results have been presented to solve the problem of local minima in multiagent systems.

This paper aims to investigate the formation control of leader-follower multiagent systems, where the problem of collision avoidance is also considered. The graph theory is used to model the communication topology between agents. To improve the control performance, a novel formation algorithm, neural-fuzzy formation controller, is proposed for multiagent systems in directed graphs. The neural-fuzzy control parameter consists of input Gaussian membership functions and output fuzzy singletons, where the parameters of input and output membership functions can be adaptively adjusted. The proposed neural-fuzzy formation controller has the capability of on-line learning, and the adaptive rules can be derived using the gradient descent method. Moreover, a fuzzy based separation control is presented for collision avoidance, and the local minimum problem of traditional potential-based separation control can be solved. The fuzzy based separation control consists of triangular input membership functions and singleton output membership functions, where the control output provides an alternative moving direction for agents to achieve collision-free tasks. Numerical simulations are provided to validate the collisionfree formation responses.

This paper is organized as follows. In Section 2, some essential graph-theoretic concepts and a network of singleintegrator agents are introduced. In Section 3, the framework of a neural-fuzzy formation controller is investigated, where the updating rules for controller parameters are derived. In Section 4, the conventional potential-based collision avoidance is introduced. Moreover, a novel fuzzy-oriented separation control is presented. In Section 5, simulation results are provided for performance validations. Some concluding remarks are given in Section 6.

\section{Preliminaries}

2.1. Graph Theory. Considering a multiagent system of $n$ agents, let $G=(V, \Xi)$ be a directed graph (digraph), consisting of a vertex set $V=\left\{v_{1}, v_{2}, \ldots, v_{n}\right\}$ and an edge set $\Xi \subseteq$ $V \times V$. The vertexes $v_{i}$ and $v_{j}$ represent the $i$ th and $j$ th agents, respectively. In digraphs, an edge of $G$ is an ordered pair of distinct nodes $\left(v_{j}, v_{i}\right) \in \Xi$, in which $v_{i}$ and $v_{j}$ are the head and tail of the edge, respectively [43]. The weighted adjacency matrix of a digraph $G$ is denoted as

$$
A=\left[\begin{array}{cccc}
a_{11} & a_{12} & \ldots & a_{1 n} \\
a_{21} & a_{22} & \ldots & a_{2 n} \\
\vdots & \vdots & \ddots & \vdots \\
a_{n 1} & a_{n 2} & \ldots & a_{n n}
\end{array}\right] \in R^{n \times n}
$$

where $a_{i j}$ is the link weight; $a_{i j}=1$, if $\left(v_{j}, v_{i}\right) \in \Xi$, and $a_{i j}=0$, if $\left(v_{j}, v_{i}\right) \notin \Xi$.

In this paper, a leader-follower problem will be dealt with, where the multiagent system consists of $n$ agents, one leader, and $n-1$ followers. In notations, the agents indexed by $1,2, \ldots, n-1$ are followers and the item $n$ is the leader. Assume that the leader agent has only transmitting capability, that is, the leader acquires no information from followers, 
$a_{n j}=0, j=1, \ldots, n$. In this case, let the topology relationship of follower agents be denoted as $\bar{G}$, a subgraph of $G$. Then, the associated adjacency matrix of $\bar{G}$ is represented as

$$
\bar{A}=\left[\begin{array}{cccc}
a_{11} & a_{12} & \ldots & a_{1(n-1)} \\
a_{21} & a_{22} & \ldots & a_{2(n-1)} \\
\vdots & \vdots & \ddots & \vdots \\
a_{(n-1) 1} & a_{(n-1) 2} & \ldots & a_{(n-1)(n-1)}
\end{array}\right] \in R^{(n-1) \times(n-1)} .
$$

Consequently, the connection relationship between the leader and followers can be described as $\bar{B}=\operatorname{diag}\left\{\bar{b}_{1}\right.$, $\left.\bar{b}_{2}, \ldots, \bar{b}_{n-1}\right\}$, where $\bar{b}_{i}=a_{i n}, i=1,2, \ldots, n-1$.

2.2. Single-Integrator Multiagent System. In this paper, a single-integrator network is considered as

$$
\dot{r}_{i}(t)=u_{i}(t),
$$

where $r_{i}(t)=\left[\begin{array}{ll}x_{i}(t) & y_{i}(t)\end{array}\right]^{T} \in R^{2}$ is the position vector and $u_{i}(t)=\left[u_{x i}(t) u_{y i}(t)\right]^{T} \in R^{2}$ is the control input vector of the $i$ th agent, $i=1,2, \ldots, n$. It is assumed that all agents have the same environment sensing capability. In addition to formation keeping, each agent is not allowed to collide with other agents during the whole moving process.

The geometric relationship between agents is shown in Figure 1 , where $R_{s}$ is the sensing radius. The node $j$ is a neighboring agent of the $i$ th node if the Euclidean distance between two agents is less or equal to the sensing radius, $d_{i j}=\left\|r_{i}-r_{j}\right\| \leq R_{s}$. Let $N_{i}$ stand for the neighbor set of the $i$ th agent. Once the $j$ th agent moves inside the sensing radius of the $i$ th agent, the collision-avoidance mechanism starts to work. In Figure 1, the notation $r_{a}$ denotes the avoidance radius of which the minimum distance allowed between two agents is $2 r_{a}$. In this case, it is reasonable that $R_{s}>2 r_{a}$ for preventing collisions.

\section{Neural Fuzzy Formation Control}

3.1. Structure of Neural Fuzzy Controller. In this section, a neural fuzzy control (NFC) is proposed to deal with the leader-following formation problem, where the single-integrator model of (3) is considered. First, let the $x$-and $y$-axis error functions be, respectively, defined as

$$
\begin{array}{r}
e_{x i}(t)=\sum_{j=1, j \neq i}^{n-1} a_{i j}\left[\left(x_{i}(t)-p_{x i}\right)-\left(x_{j}(t)-p_{x j}\right)\right] \\
+\bar{b}_{i}\left[\left(x_{i}(t)-p_{x i}\right)-\left(x_{n}(t)-p_{x n}\right)\right], \\
e_{y i}(t)=\sum_{j=1, j \neq i}^{n-1} a_{i j}\left[\left(y_{i}(t)-p_{y i}\right)-\left(y_{j}(t)-p_{y j}\right)\right] \\
+\bar{b}_{i}\left[\left(y_{i}(t)-p_{y i}\right)-\left(y_{n}(t)-p_{y n}\right)\right],
\end{array}
$$

where $p_{x i}, p_{x n}, p_{y i}$, and $p_{y n}$ are coordinate positions regarding a desired formation pattern in $x$ - and $y$-axes. It is noticed that $a_{i j}=1$ means that the $j$ th agent can send position
TABLe 1: Fuzzy rule base.

\begin{tabular}{lllllll}
\hline$S_{x i}\left(S_{y i}\right)$ & $\mathrm{NB}$ & $\mathrm{NM}$ & $\mathrm{NS}$ & $\mathrm{PS}$ & $\mathrm{PM}$ & $\mathrm{PB}$ \\
\hline$u_{x i}\left(u_{y i}\right)$ & $\mathrm{PB}$ & $\mathrm{PM}$ & $\mathrm{PS}$ & $\mathrm{NS}$ & $\mathrm{NM}$ & $\mathrm{NB}$ \\
\hline
\end{tabular}

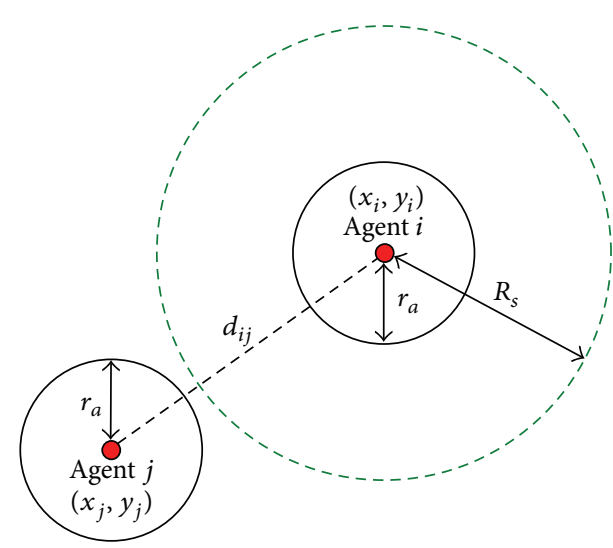

(a)

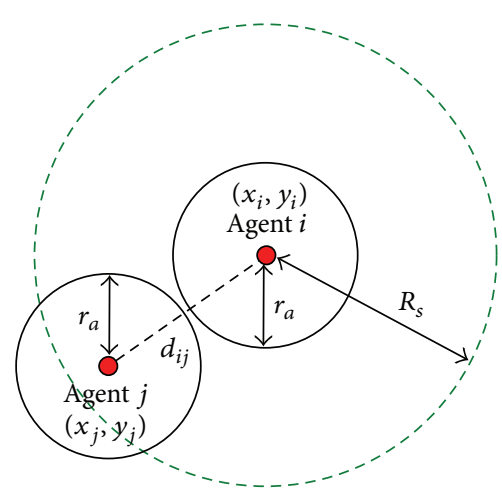

(b)

FIGURE 1: Geometric relationship between agents: (a) node $j$ is not a neigh-boring agent of node $i$; (b) node $j$ is a neighboring agent of node $i$.

information to the $i$ th agent, and $\bar{b}_{i}=1$ means that the $i$ th agent can receive position information from the leader. In this study, the leader is maneuvered along a prespecified trajectory, and the design of formation controller is focused on followers. Let the controller inputs of the $i$ th follower agent be designated as follows

$$
\begin{aligned}
& S_{x i}=c_{1} \cdot e_{x i}+c_{2} \cdot \int_{0}^{t} e_{x i} d t, \\
& S_{y i}=c_{1} \cdot e_{y i}+c_{2} \cdot \int_{0}^{t} e_{y i} d t,
\end{aligned}
$$

where $c_{1}$ and $c_{2}$ are positive constants, $i=1,2, \ldots, n-1$.

The network structure of NFC is shown in Figure 2. The fuzzy rules are given in Table 1 , where the input and output spaces are fuzzily partitioned into six fuzzy sets, Negative Big (NB), Negative Medium (NM), Negative Small (NS), Positive Small (PS), Positive Medium (PM), and Positive Big (PB). The input and output membership functions are depicted in 


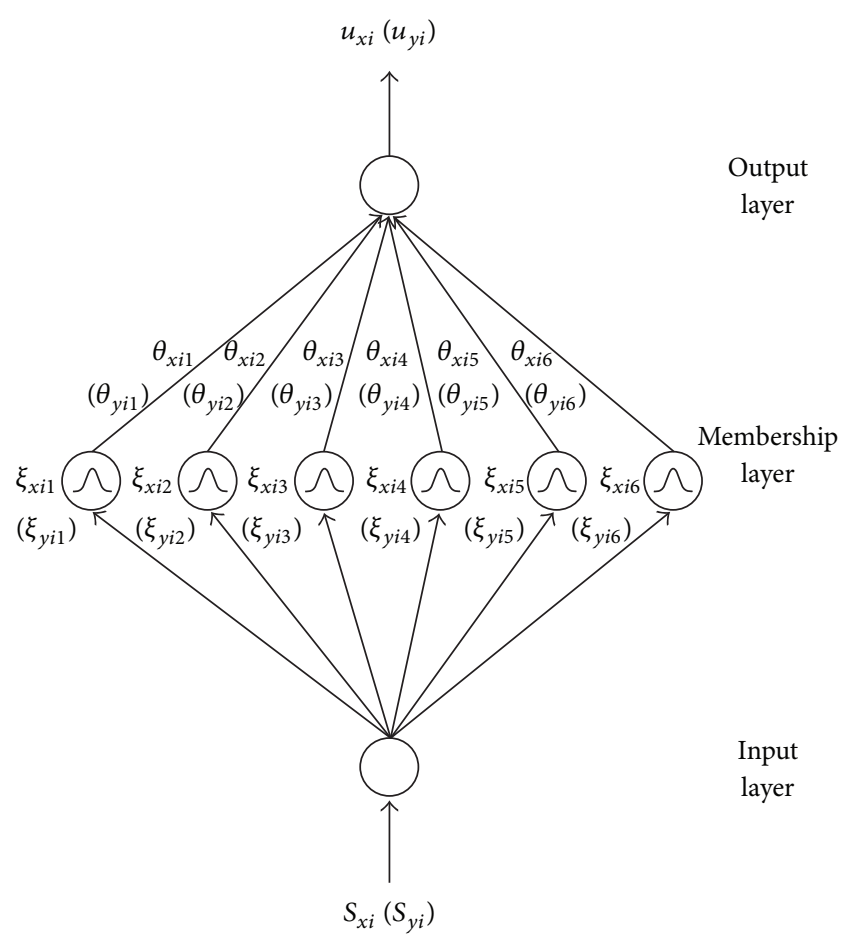

Figure 2: Structure of NFC.

Figure 3. The corresponding if-then fuzzy rules for the $i$ th agent are expressed as

$$
\begin{aligned}
R_{i k}: & \operatorname{IF} S_{x i}\left(S_{y i}\right) \text { is } \xi_{x i k}\left(\xi_{y i k}\right) \\
& \operatorname{THEN} u_{x i}\left(u_{y i}\right) \text { is } \theta_{x i k}\left(\theta_{y i k}\right),
\end{aligned}
$$

where $\xi_{x i k}\left(\xi_{y i k}\right)$ and $\theta_{x i k}\left(\theta_{y i k}\right)$ are the fuzzy sets of the antecedent and consequence parts, respectively, $i=1,2, \ldots$, $n-1, k=1,2, \ldots, 6$. In Figure 3 , the $k$ th nodes of the membership layer are Gaussian functions,

$$
\begin{aligned}
& \xi_{x i k}\left(S_{x i}, \sigma_{x i}, C_{x i}\right)=\exp \left[\frac{-1}{2}\left(\frac{S_{x i}-c_{x i k}}{\sigma_{x i k}}\right)^{2}\right], \\
& \xi_{y i k}\left(S_{y i}, \sigma_{y i}, C_{y i}\right)=\exp \left[\frac{-1}{2}\left(\frac{S_{y i}-c_{y i k}}{\sigma_{y i k}}\right)^{2}\right],
\end{aligned}
$$

where

$$
\begin{aligned}
\sigma_{x i} & =\left[\begin{array}{llllll}
\sigma_{x i 1} & \sigma_{x i 2} & \sigma_{x i 3} & \sigma_{x i 4} & \sigma_{x i 5} & \sigma_{x i 6}
\end{array}\right]^{T}, \\
\sigma_{y i} & =\left[\begin{array}{llllll}
\sigma_{y i 1} & \sigma_{y i 2} & \sigma_{y i 3} & \sigma_{y i 4} & \sigma_{y i 5} & \sigma_{y i 6}
\end{array}\right]^{T}, \\
C_{x i} & =\left[\begin{array}{lllllll}
c_{x i 1} & c_{x i 2} & c_{x i 3} & c_{x i 4} & c_{x i 5} & c_{x i 6}
\end{array}\right]^{T}, \\
C_{y i} & =\left[\begin{array}{lllllll}
c_{y i 1} & c_{y i 2} & c_{y i 3} & c_{y i 4} & c_{y i 5} & c_{y i 6}
\end{array}\right]^{T},
\end{aligned}
$$

$i=1,2, \ldots, n-1$. In (8) and (9), $c_{x i k}\left(c_{y i k}\right)$ and $\sigma_{x i k}\left(\sigma_{y i k}\right)$ are means and standard deviations, respectively, $k=1,2, \ldots, 6$.

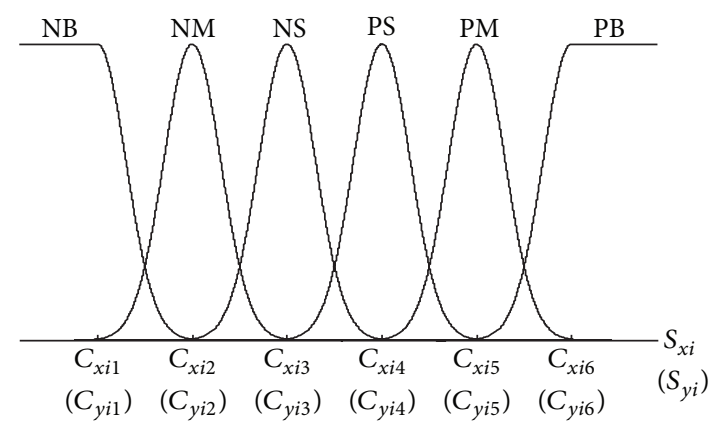

(a)

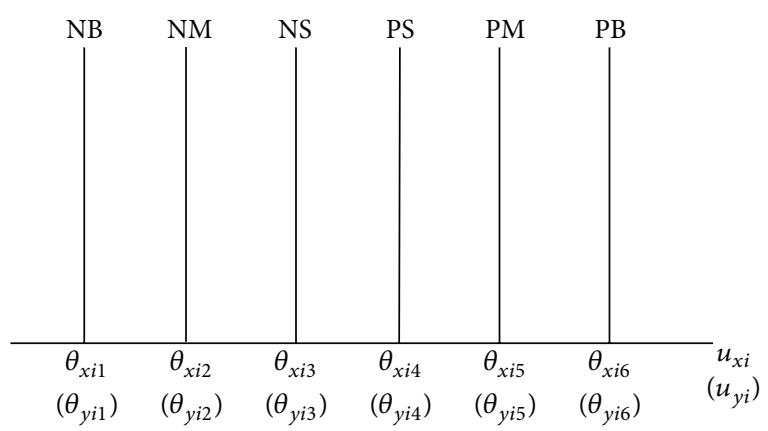

(b)

FIGURE 3: Membership functions of NFC.

By using the centroid defuzzification technique, the NFC output can be calculated as follows:

$$
\begin{aligned}
& u_{x i}\left(S_{x i}, \xi_{x i}, \theta_{x i}\right)=\frac{\sum_{k=1}^{6} \xi_{x i k} \theta_{x i k}}{\sum_{k=1}^{6} \xi_{x i k}}=\theta_{x i}^{T} \xi_{x i}, \\
& u_{y i}\left(S_{y i}, \xi_{y i}, \theta_{y i}\right)=\frac{\sum_{k=1}^{6} \xi_{y i k} \theta_{y i k}}{\sum_{k=1}^{6} \xi_{y i k}}=\theta_{y i}^{T} \xi_{y i},
\end{aligned}
$$

where $\theta_{x i k}\left(\theta_{y i k}\right)$ are the values corresponding to singleton outputs,

$$
\begin{aligned}
& \theta_{x i}=\left[\begin{array}{llllll}
\theta_{x i 1} & \theta_{x i 2} & \theta_{x i 3} & \theta_{x i 4} & \theta_{x i 5} & \theta_{x i 6}
\end{array}\right]^{T}, \\
& \theta_{y i}=\left[\begin{array}{llllll}
\theta_{y i 1} & \theta_{y i 2} & \theta_{y i 3} & \theta_{y i 4} & \theta_{y i 5} & \theta_{y i 6}
\end{array}\right]^{T},
\end{aligned}
$$

$$
\begin{aligned}
& \xi_{x i}=\left[\begin{array}{llllll}
\frac{\xi_{x i 1}}{\xi_{x i}^{\prime}} & \frac{\xi_{x i 2}}{\xi_{x i}^{\prime}} & \frac{\xi_{x i 3}}{\xi_{x i}^{\prime}} & \frac{\xi_{x i 4}}{\xi_{x i}^{\prime}} & \frac{\xi_{x i 5}}{\xi_{x i}^{\prime}} & \frac{\xi_{x i 6}}{\xi_{x i}^{\prime}}
\end{array}\right]^{T}, \\
& \xi_{y i}=\left[\begin{array}{llllll}
\frac{\xi_{y i 1}}{\xi_{y i}^{\prime}} & \frac{\xi_{y i 2}}{\xi_{y i}^{\prime}} & \frac{\xi_{y i 3}}{\xi_{y i}^{\prime}} & \frac{\xi_{y i 4}}{\xi_{y i}^{\prime}} & \frac{\xi_{y i 5}}{\xi_{y i}^{\prime}} & \frac{\xi_{y i 6}}{\xi_{y i}^{\prime}}
\end{array}\right]^{T},
\end{aligned}
$$

in which $\xi_{x i}^{\prime}=\sum_{k=1}^{6} \xi_{x i k}$ and $\xi_{y i}^{\prime}=\sum_{k=1}^{6} \xi_{y i k}$.

3.2. Parameter-Learning Algorithms. In this section, the idea of gradient descent will be adopted to derive on-line learning 


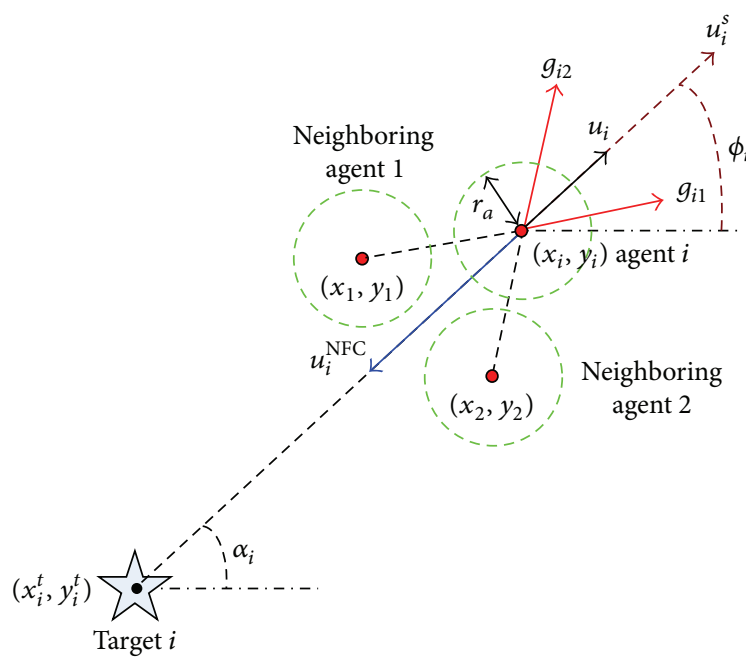

FIgURE 4: Schematic diagram of local minima problem.

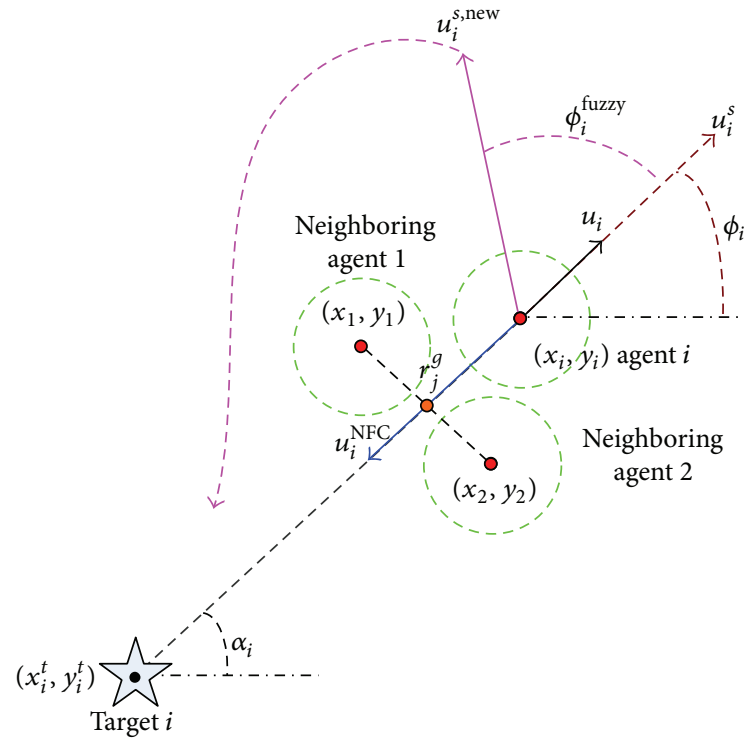

FIGURE 5: Schematic diagram of improved avoidance functions.

algorithms to update NFC parameters. First, energy functions $E_{x i}$ and $E_{y i}$ are defined as follows:

$$
\begin{aligned}
& E_{x i}=\frac{1}{2}\left(S_{x i}\right)^{2}, \\
& E_{y i}=\frac{1}{2}\left(S_{y i}\right)^{2},
\end{aligned}
$$

where $i=1,2, \ldots, n-1$. Then, the update laws of layer parameters are described in the following.

(1) Output Layer. According to the gradient decent method [44], $\theta_{x i k}$ and $\theta_{y i k}$ are updated by the following rules:

$$
\begin{aligned}
& \Delta \theta_{x i k}=-\eta_{1} \frac{\partial E_{x i}}{\partial \theta_{x i k}}=-\eta_{1} \frac{\partial E_{x i}}{\partial u_{x i}} \frac{\partial u_{x i}}{\partial \theta_{x i k}}, \\
& \Delta \theta_{y i k}=-\eta_{1} \frac{\partial E_{y i}}{\partial \theta_{y i k}}=-\eta_{1} \frac{\partial E_{y i}}{\partial u_{y i}} \frac{\partial u_{y i}}{\partial \theta_{y i k}}
\end{aligned}
$$

where $\eta_{1}>0$ is a learning rate, $i=1,2, \ldots, n-1, k=$ $1,2, \ldots, 6$. From (11), it can be obtained that

$$
\begin{aligned}
& \frac{\partial u_{x i}}{\partial \theta_{x i k}}=\frac{\xi_{x i k}}{\sum_{k=1}^{6} \xi_{x i k}}, \\
& \frac{\partial u_{y i}}{\partial \theta_{y i k}}=\frac{\xi_{y i k}}{\sum_{k=1}^{6} \xi_{y i k}} .
\end{aligned}
$$

In (14), the error term to be propagated can be reformulated as

$$
\begin{aligned}
& \frac{\partial E_{x i}}{\partial u_{x i}}=\frac{\partial E_{x i}}{\partial S_{x i}} \frac{\partial S_{x i}}{\partial u_{x i}}, \\
& \frac{\partial E_{y i}}{\partial u_{y i}}=\frac{\partial E_{y i}}{\partial S_{y i}} \frac{\partial S_{y i}}{\partial u_{y i}} .
\end{aligned}
$$

However, the terms $\partial S_{x i} / \partial u_{x i}$ and $\partial S_{y i} / \partial u_{y i}$ in (16) cannot be analytically determined. To overcome this problem, the following adaptive laws are adopted [45]:

$$
\begin{aligned}
& -\frac{\partial E_{x i}}{\partial u_{x i}} \approx k_{1} \cdot S_{x i}+k_{2} \cdot \dot{S}_{x i}, \\
& -\frac{\partial E_{y i}}{\partial u_{y i}} \approx k_{1} \cdot S_{y i}+k_{2} \cdot \dot{S}_{y i},
\end{aligned}
$$

where $k_{1}$ and $k_{2}$ are positive constants.

In summary, from (15) and (17), the parameters of the output layer can be adaptively updated.

(2) Membership Layer. The parameters $c_{x i k}$ and $c_{y i k}$ are updated by the following rules:

$$
\begin{gathered}
\Delta c_{x i k}=-\eta_{2} \frac{\partial E_{x i}}{\partial c_{x i k}}=-\eta_{2} \frac{\partial E_{x i}}{\partial u_{x i}} \frac{\partial u_{x i}}{\partial \xi_{x i k}} \frac{\partial \xi_{x i k}}{\partial c_{x i k}}, \\
\Delta c_{y i k}=-\eta_{2} \frac{\partial E_{y i}}{\partial c_{y i k}}=-\eta_{2} \frac{\partial E_{y i}}{\partial u_{y i}} \frac{\partial u_{y i}}{\partial \xi_{y i k}} \frac{\partial \xi_{y i k}}{\partial c_{y i k}},
\end{gathered}
$$

where $\eta_{2}>0$ is a learning rate, $i=1,2, \ldots, n-1, k=$ $1,2, \ldots, 6$. From (8), (9), and (11), it can be obtained that

$$
\begin{aligned}
\frac{\partial u_{x i}}{\partial \xi_{x i k}} \frac{\partial \xi_{x i k}}{\partial c_{x i k}}= & \frac{\theta_{x i k} \cdot\left(\sum_{k=1}^{6} \xi_{x i k}\right)-\sum_{k=1}^{6} \xi_{x i k} \theta_{x i k}}{\left(\sum_{k=1}^{6} \xi_{x i k}\right)^{2}} \\
& \cdot \exp \left[\frac{-1}{2}\left(\frac{S_{x i}-c_{x i k}}{\sigma_{x i k}}\right)^{2}\right] \cdot\left(\frac{S_{x i}-c_{x i k}}{\sigma_{x i k}^{2}}\right), \\
\frac{\partial u_{y i}}{\partial \xi_{y i k}} \frac{\partial \xi_{y i k}}{\partial c_{y i k}}= & \frac{\theta_{y i k} \cdot\left(\sum_{k=1}^{6} \xi_{y i k}\right)-\sum_{k=1}^{6} \xi_{y i k} \theta_{y i k}}{\left(\sum_{k=1}^{6} \xi_{y i k}\right)^{2}} \\
& \cdot \exp \left[\frac{-1}{2}\left(\frac{S_{y i}-c_{y i k}}{\sigma_{y i k}}\right)^{2}\right] \cdot\left(\frac{S_{y i}-c_{y i k}}{\sigma_{y i k}^{2}}\right) .
\end{aligned}
$$


TABLe 2: Fuzzy rule base.

\begin{tabular}{lcccccccccccccccccccccccccc}
\hline Rule & 1 & 2 & 3 & 4 & 5 & 6 & 7 & 8 & 9 & 10 & 11 & 12 & 13 & 14 & 15 & 16 & 17 & 18 & 19 & 20 & 21 & 22 & 23 & 24 & 25 \\
\hline$z_{i}^{1}$ & NB & NB & NB & NB & NB & NS & NS & NS & NS & NS & ZO & ZO & ZO & ZO & ZO & PS & PS & PS & PS & PS & PB & PB & PB & PB & PB \\
\hline$z_{i}^{2}$ & VC & C & M & F & VF & VC & C & M & F & VF & VC & C & M & F & VF & VC & C & M & F & VF & VC & C & M & F & VF \\
\hline$\phi_{i}^{\text {fuzzy }}$ & PS & PS & PS & ZO & ZO & PB & PB & PS & PS & PS & PB & PB & PB & PB & PB & PB & PB & PS & PS & PS & PS & PS & PS & ZO & ZO \\
\hline
\end{tabular}

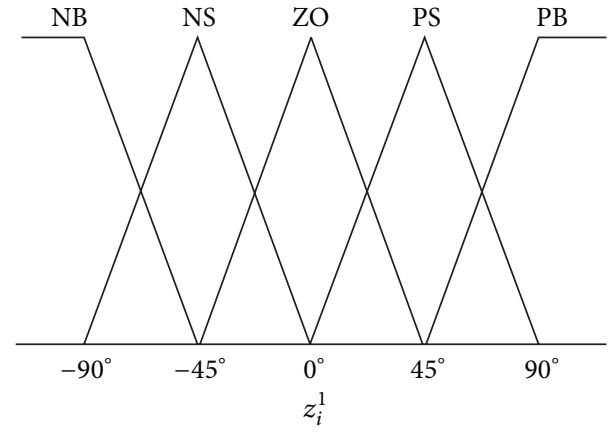

(a)

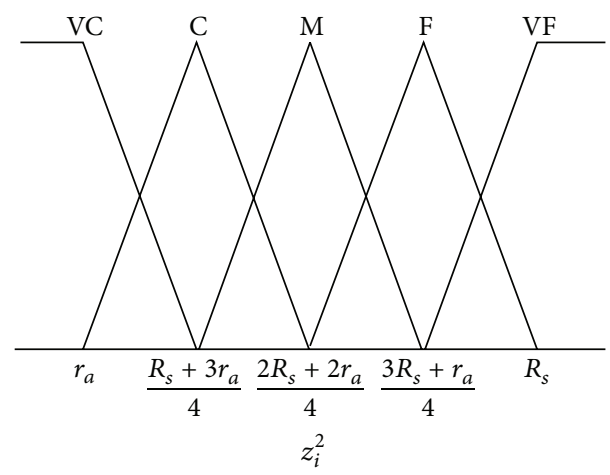

(b)

FIgURE 6: The input membership functions.

Similarly, $\sigma_{x i k}$ and the $\sigma_{y i k}$ are updated by the following amounts:

$$
\begin{gathered}
\Delta \sigma_{x i k}=-\eta_{3} \frac{\partial E_{x i}}{\partial \sigma_{x i k}}=-\eta_{3} \frac{\partial E_{x i}}{\partial u_{x i}} \frac{\partial u_{x i}}{\partial \xi_{x i k}} \frac{\partial \xi_{x i k}}{\partial \sigma_{x i k}}, \\
\Delta \sigma_{y i k}=-\eta_{3} \frac{\partial E_{y i}}{\partial \sigma_{y i k}}=-\eta_{3} \frac{\partial E_{y i}}{\partial u_{y i}} \frac{\partial u_{y i}}{\partial \xi_{y i k}} \frac{\partial \xi_{y i k}}{\partial \sigma_{y i k}}
\end{gathered}
$$

where $\eta_{3}>0$ is a learning rate, $i=1,2, \ldots, n, k=1,2, \ldots, 6$. From (8), (9), and (11), it can be obtained that

$$
\begin{aligned}
\frac{\partial u_{x i}}{\partial \xi_{x i k}} \frac{\partial \xi_{x i k}}{\partial \sigma_{x i k}}= & \frac{\theta_{x i k} \cdot\left(\sum_{k=1}^{6} \xi_{x i k}\right)-\sum_{k=1}^{6} \xi_{x i k} \theta_{x i k}}{\left(\sum_{k=1}^{6} \xi_{x i k}\right)^{2}} \\
& \cdot \exp \left[\frac{-1}{2}\left(\frac{S_{x i}-c_{x i k}}{\sigma_{x i k}}\right)^{2}\right] \cdot \frac{\left(S_{x i}-c_{x i k}\right)^{2}}{\left(\sigma_{x i k}\right)^{3}},
\end{aligned}
$$

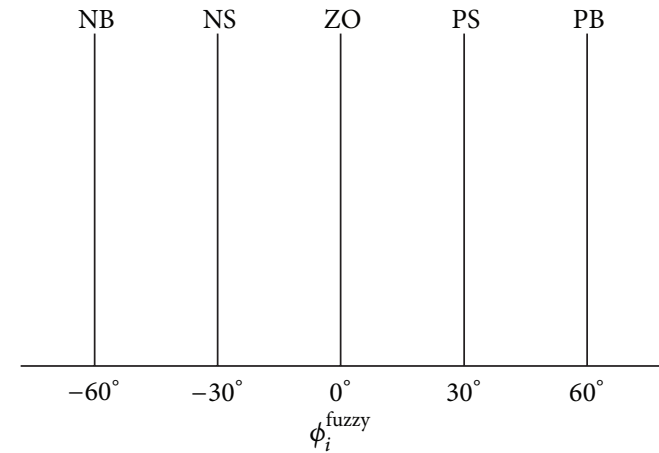

FIgURE 7: The output membership functions.

$$
\begin{aligned}
\frac{\partial u_{y i}}{\partial \xi_{y i k}} \frac{\partial \xi_{y i k}}{\partial \sigma_{y i k}}= & \frac{\theta_{y i k} \cdot\left(\sum_{k=1}^{6} \xi_{y i k}\right)-\sum_{k=1}^{6} \xi_{y i k} \theta_{y i k}}{\left(\sum_{k=1}^{6} \xi_{y i k}\right)^{2}} \\
& \cdot \exp \left[\frac{-1}{2}\left(\frac{S_{y i}-c_{y i k}}{\sigma_{y i k}}\right)^{2}\right] \cdot \frac{\left(S_{y i}-c_{y i k}\right)^{2}}{\left(\sigma_{y i k}\right)^{3}} .
\end{aligned}
$$

In summary, from (18)-(21), the parameters of the input layer can be adaptively updated.

\section{Separation Control for Collision Avoidance}

4.1. Potential-Based Separation Control. Let $g_{i j}$ be the separation force between the $i$ th and $j$ th agents. Then, the integrated separation force from all its neighboring agents can be formulated as

$$
u_{i}^{s}=-\sum_{j \in N_{i}} g_{i j}\left(r_{i}, r_{j}\right)
$$

To derive a proper separation force between two connected agents, a smooth potential function $V_{i j}$ is considered,

$$
V_{i j}\left(d_{i j}\right)=\left[\min \left(0, \frac{d_{i j}^{2}-R_{s}^{2}}{d_{i j}^{2}-4 r_{a}^{2}}\right)\right]^{2},
$$

such that

$$
g_{i j}=\nabla_{r_{i}} V_{i j}
$$



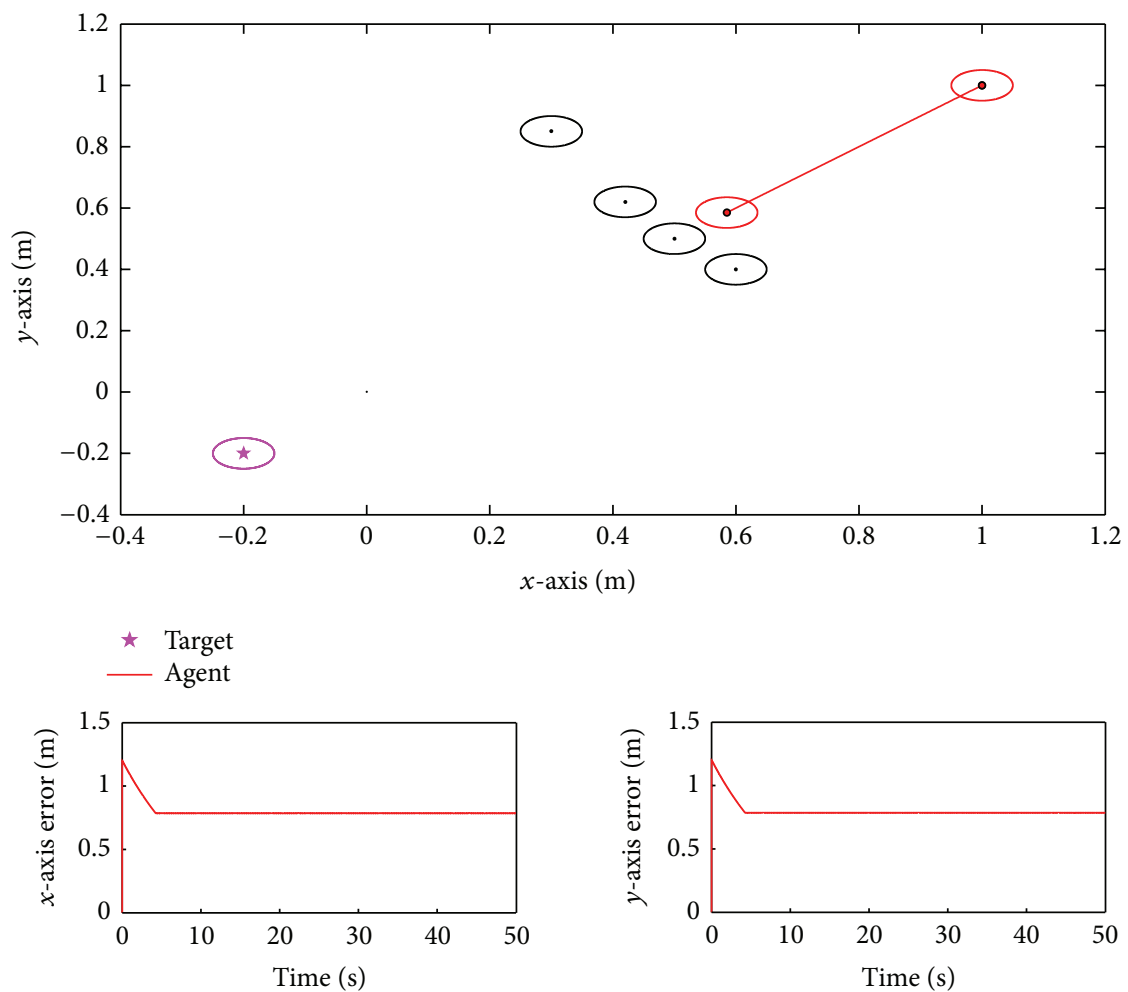

(a)
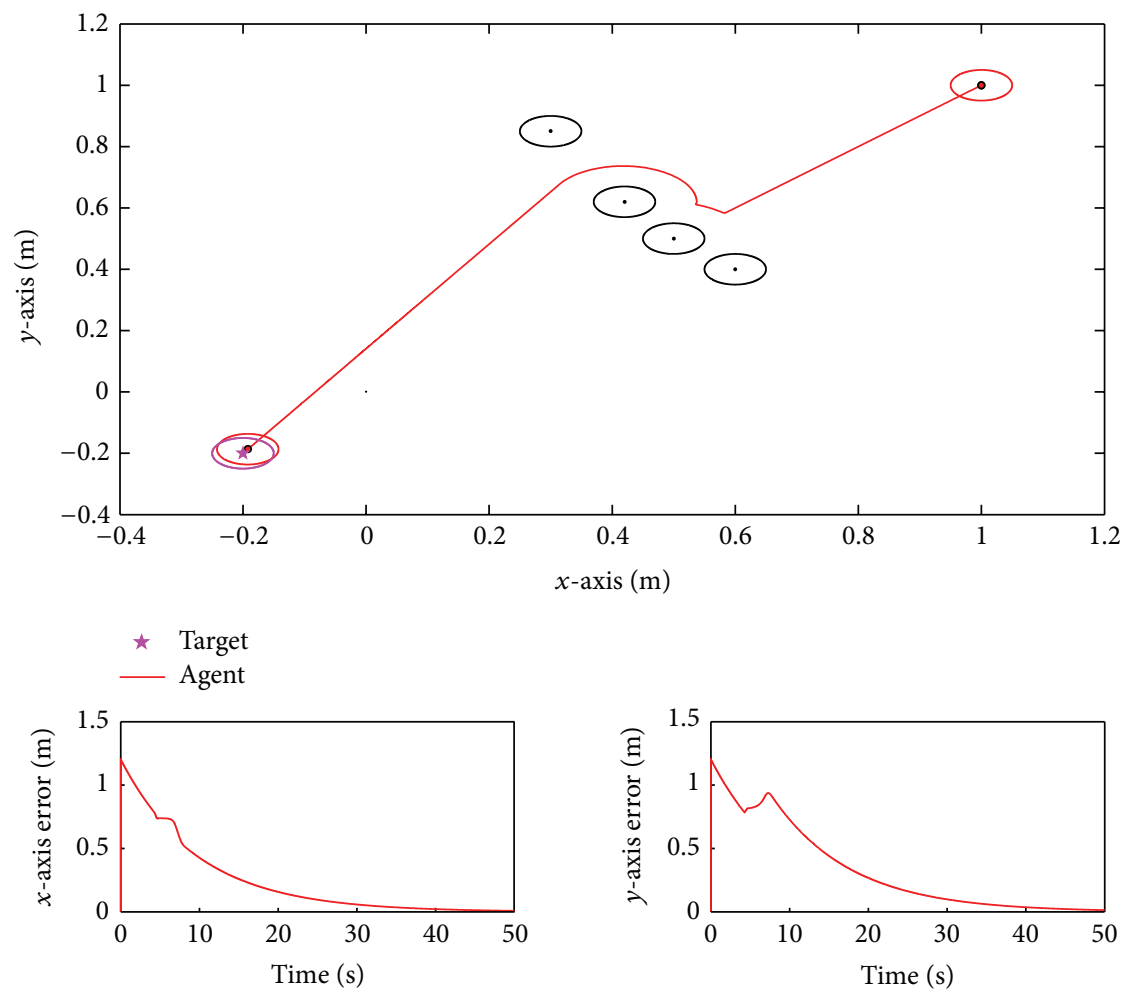

(b)

FIGURE 8: Simulation results of collision avoidance: (a) linger in local minimum (PSC) and (b) bypass neighboring agents (FSC). 
In a two-dimensional case, $r_{i}=\left[\begin{array}{ll}x_{i} & y_{i}\end{array}\right]^{T}$, the gradient computations of $V_{i j}$ can be obtained as follows:

$$
\begin{gathered}
\frac{\partial V_{i j}}{\partial x_{i}}= \begin{cases}0, & \text { if } R_{s} \leq d_{i j}, \\
V_{g i j}\left(x_{i}-x_{j}\right), & \text { if } 2 r_{a}<d_{i j}<R_{s}, \\
V_{\max }, & \text { if } d_{i j}<2 r_{a},\end{cases} \\
\frac{\partial V_{i j}}{\partial y_{i}}= \begin{cases}0, & \text { if } R_{s} \leq d_{i j}, \\
V_{g i j}\left(y_{i}-y_{j}\right), & \text { if } 2 r_{a}<d_{i j}<R_{s}, \\
V_{\max }, & \text { if } d_{i j}<2 r_{a},\end{cases} \\
V_{g i j}=\frac{4\left(R_{s}^{2}-4 r_{a}^{2}\right)\left(d_{i j}^{2}-R_{s}^{2}\right)}{\left(d_{i j}^{2}-4 r_{a}^{2}\right)^{3}},
\end{gathered}
$$

where $V_{\max }$ is the maximum allowable separation force. Integrating the formation and separation forces, the net control action to an agent can be obtained as

$$
u_{i}=u_{i}^{\mathrm{NFC}}+u_{i}^{s}
$$

where $u_{i}^{\mathrm{NFC}}=\left[\begin{array}{ll}u_{x i} & u_{y i}\end{array}\right]^{T}$ is the neural fuzzy formation action.

4.2. Fuzzy Separation Control. In a multiagent system, an agent could be standstill or move back and forth if the net force acting on this agent is balanced. This phenomenon is known as the local minimum problem. In Figure 4, the case of agent $i$ with two neighboring agents is considered, where $\left(x_{1}, y_{1}\right)$ and $\left(x_{2}, y_{2}\right)$ are neighboring agents, and the related target is located at $\left(x_{i}^{t}, y_{i}^{t}\right)$. The notation $u_{i}^{\mathrm{NFC}}$ is denoted as the attractive force to the target, and $g_{i 1}$ and $g_{i 2}$ are separation forces corresponding to neighboring agents. Then, the integrated separation force to the $i$ th agent is the vector sum of $g_{i 1}$ and $g_{i 2}$, where $\phi_{i}$ is the related direction of $u_{i}^{s}$ to the $x$-axis. In addition, $u_{i}$ is the net force of the $i$ th agent, and $\alpha_{i}$ is angle between the agent $i$ and its target. In case $\phi_{i}=\alpha_{i}$, the direction of $u_{i}^{s}$ is opposite to the attractive action $u_{i}^{\mathrm{NFC}}$. Moreover, if the magnitude of $u_{i}^{\mathrm{NFC}}$ is less or equal to the magnitude of $u_{i}^{s}$, the $i$ th agent will be stuck in the local minimum. To solve this problem, a fuzzy separation control method will be presented, and the key idea is depicted in Figure 5, where an extra angle $\phi_{i}^{\text {fuzzy }}$ is added to the original separation force, $\phi_{i}^{\text {new }}=\phi_{i}+\phi_{i}^{\text {fuzzy }}$. In Figure $5, u_{i}^{s, \text { new }}$ is the modified separation force, of which the magnitude keeps unchanged but the direction is changed because of $\phi_{i}^{\text {fuzzy }}$. Consequently, the integrated net force of attractive force and separation force can be represented as

$$
u_{i}=u_{i}^{\mathrm{NFC}}+u_{i}^{s, \text { new }}
$$

Basically, $u_{i}^{s, n e w}$ can provide a new route to bypass the neighboring agents when a local minimum situation happens. It is noticed that the target of a follower is its temporary destination for next movement during the process of avoiding collision. For those follower agents, communication-connected to the leader, their respective targets can be obtained according to the leader position and designated formation pattern; however, a substitute solution is required for other followers. Alternatively, from (4) and (5), targets can be equivalently viewed as the propagated errors from other followers,

$$
\begin{aligned}
& x_{i}^{t}=x_{i}-e_{x i}, \\
& y_{i}^{t}=y_{i}-e_{y i},
\end{aligned}
$$

where follower $i$ is not communicated to the leader, $\bar{b}_{i}=0$, $i=1,2, \ldots, n-1$.

The design of fuzzy separation controller will be illustrated in the following. First, let the fuzzy inputs of the $i$ th agent be

$$
\begin{gathered}
z_{i}^{1}=\phi_{i}-\alpha_{i}, \\
z_{i}^{2}=\left\|r_{i}-r_{j}^{g}\right\|,
\end{gathered}
$$

where $r_{j}^{g}=\left[\begin{array}{ll}x_{j}^{g} & y_{j}^{g}\end{array}\right]^{T} \in R^{2}$ is the position of the center gravity of neighboring agents,

$$
\begin{aligned}
& x_{j}^{g}=\frac{\sum_{j \in N_{i}} x_{j}}{\left|N_{i}\right|}, \\
& y_{j}^{g}=\frac{\sum_{j \in N_{i}} y_{j}}{\left|N_{i}\right|},
\end{aligned}
$$

where $|\cdot|$ is the cardinality of a set, that is, $\left|N_{i}\right|$ is the number of neighboring agents of the $i$ th agent.

The fuzzy rules are given in Table 2, where the input and output spaces are fuzzily partitioned into ten fuzzy sets, Negative Big (NB), Negative Small (NS), Zero (ZO), Positive Small (PS), Positive Big (PB), Very Close (VC), Close (C), Medium (M), Far (F), and Very Far (VF). The input and output membership functions are depicted in Figures 6 and 7 , respectively. The corresponding if-then fuzzy rules for the $i$ th agent are expressed as

$$
R_{i}: \operatorname{IF} z_{i}^{1} \text { is } M_{i k}^{1} \operatorname{AND} z_{i}^{2} \text { is } M_{i k}^{2} \text { THEN } \phi_{i}^{\mathrm{fuzzy}} \text { is } G_{i k} \text {, }
$$

where $M_{i k}^{1}$ and $M_{i k}^{2}$ are the fuzzy sets of the antecedent part, and $G_{i k}$ is the fuzzy set of the consequence part, $i=1,2, \ldots$, $n-1$, and $k=1,2, \ldots, 5$. By using the centroid defuzzification technique, the defuzzified fuzzy output is calculated as

$$
\phi_{i}^{\mathrm{fuzzy}}=\frac{\sum_{k=1}^{5} \mu_{i k}\left(G_{i k}\right) \cdot G_{i k}}{\sum_{k=1}^{5} \mu_{i k}\left(G_{i k}\right)},
$$

where a min-max operation is performed over all rules mapping to the same output fuzzy set:

$$
\mu_{i k}\left(G_{i k}\right)=\max _{G_{i k}}\left\{\min \left(\mu_{M_{i k}^{1}}\left(G_{i k}\right), \mu_{M_{i k}^{2}}\left(G_{i k}\right)\right)\right\} .
$$



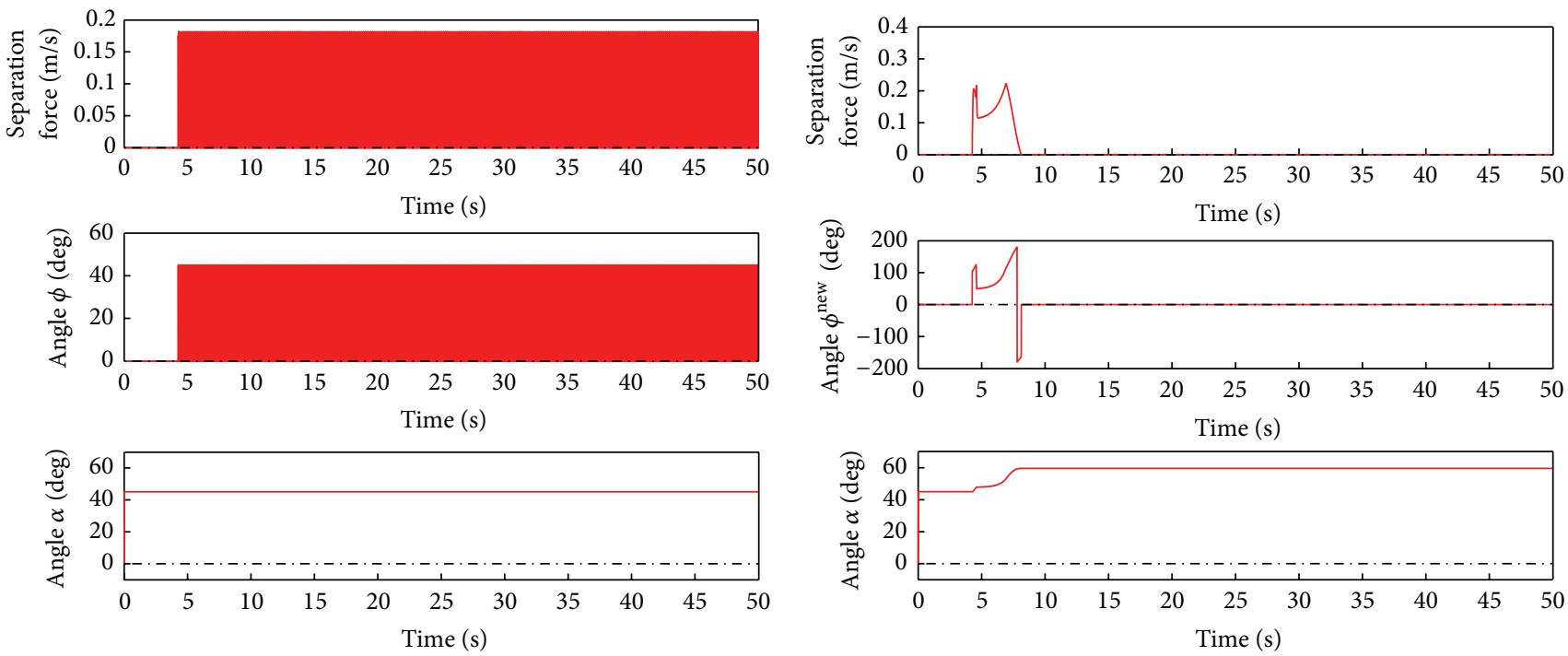

(a)

(b)

FIgURE 9: Separation force and angle of collision avoidance: (a) PSC and (b) FSC.

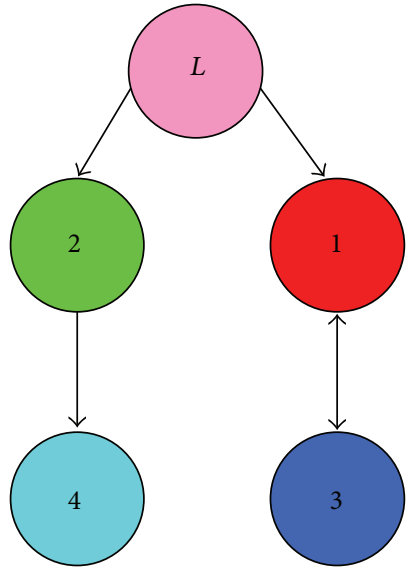

Figure 10: Communication topology of multiagent systems.

\section{Simulation Results}

In the following, all the agents are assumed to be homogeneous with the same specifications, $r_{a}=0.05(\mathrm{~m})$ and $R_{s}=0.15(\mathrm{~m})$. To verify the feasibility of proposed neurofuzzy formation controller and fuzzy separation controller, both the collision avoidance and formation preservation are considered.

5.1. Collision Avoidance with Static Target. One dynamical agent is initially placed at the point $(1,1)(\mathrm{m})$, and four fixed agents are located at $(0.5,0.5),(0.6,0.4),(0.42,0.62)$, and $(0.3,0.85)(\mathrm{m})$, respectively. The target position is given as $(-0.2,-0.2)(\mathrm{m})$. It is desired that the dynamic agent can reach the designated target without colliding with fixed agents. Figures 8 and 9 illustrate the collision-avoidance responses corresponding to potential-based separation control (PSC) and the proposed fuzzy separation control (FSC), where the formation control of each case is the conventional consensus algorithm [46]. In Figure 8, it can be seen that the agent lingers in front of neighboring agents with PSC. On the other hand, the agent can successfully bypass neighboring agents with the proposed FSC. The responses of position errors, shown in the bottom two subplots of Figure 8, indicate that the desired target can be asymptotically achieved with the proposed FSC. However, a local-minimum behavior exists by using the PSC, and the related separation force keeps oscillation. The oscillation behavior of the separation force is coincided with the response of $\phi$, shown in Figure 9(a). In Figure 9(b), the angle $\phi=0$ after 8.26 (sec) means that the dynamic agent successfully bypasses the fixed agents, and thus, there is no separation force.

5.2. Formation Control and Collision Avoidance with Static Leader. In leader-follower formation control, the case of five agents, one static leader and four followers, is considered. The communication topology is shown in Figure 10, where the circles labelled 1 to 4 denote the follower agents and the circle $L$ represents the leader agent. From (2), the information exchanges between leader and followers can be modelled as

$$
\bar{A}=\left[\begin{array}{llll}
0 & 0 & 1 & 0 \\
0 & 0 & 0 & 0 \\
1 & 0 & 0 & 0 \\
0 & 1 & 0 & 0
\end{array}\right], \quad \bar{B}=\left[\begin{array}{llll}
1 & 0 & 0 & 0 \\
0 & 1 & 0 & 0 \\
0 & 0 & 0 & 0 \\
0 & 0 & 0 & 0
\end{array}\right]
$$

The followers are initially placed at the points $(0,-0.25)$, $(0.25,0),(0,0.25)$, and $(-0.25,0)(\mathrm{m})$, respectively, and the position of the leader is $(0.5,0)(\mathrm{m})$. The formation pattern is designated as $\left(p_{x 1}, p_{y 1}\right)=(-0.5,0.25),\left(p_{x 2}, p_{y 2}\right)=$ $(-0.75,0),\left(p_{x 3}, p_{y 3}\right)=(-0.5,-0.25),\left(p_{x 4}, p_{y 4}\right)=(-0.25,0)$, and $\left(p_{x 5}, p_{y 5}\right)=(0,0)(\mathrm{m})$. The parameters of the NFC are 

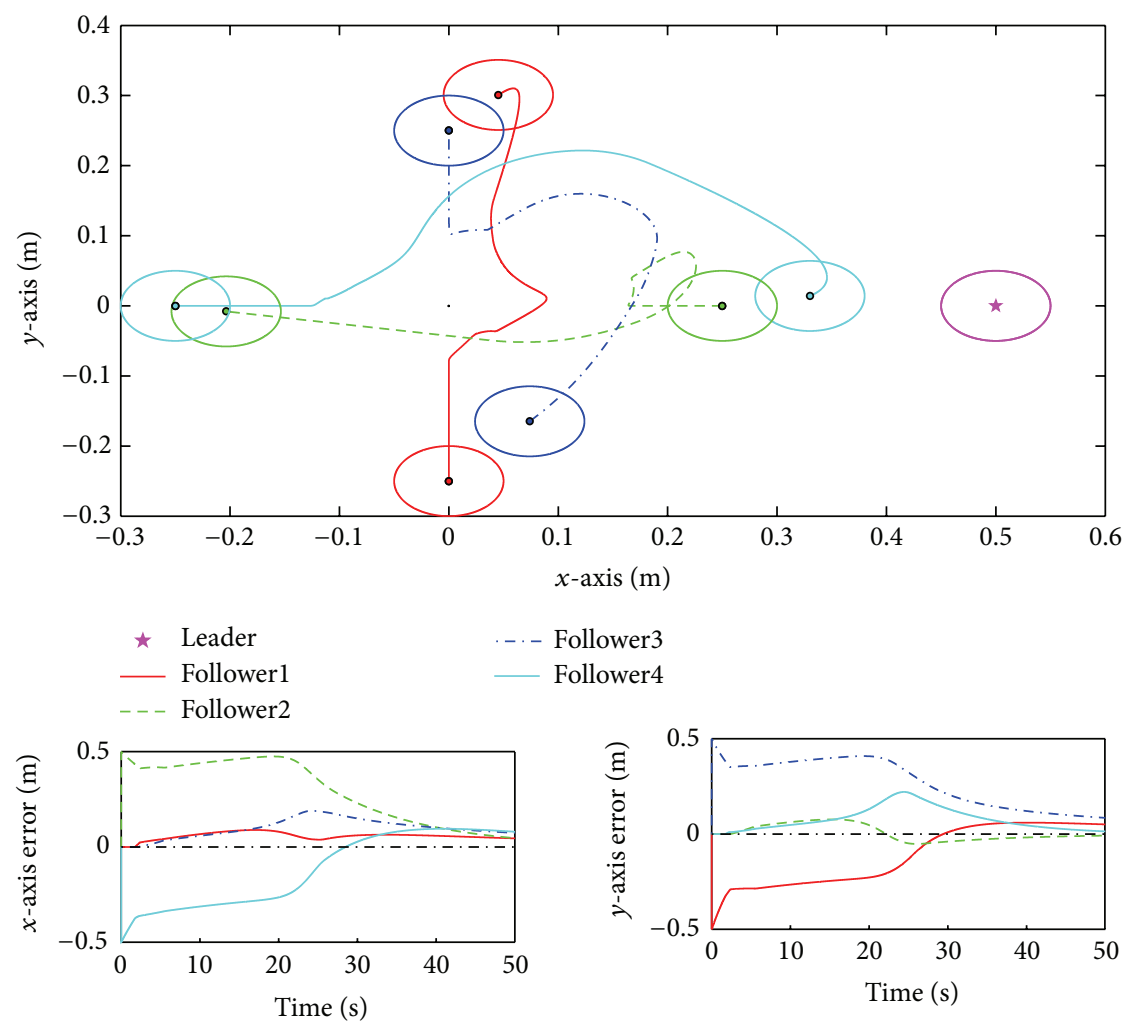

-. - Follower3

Follower4

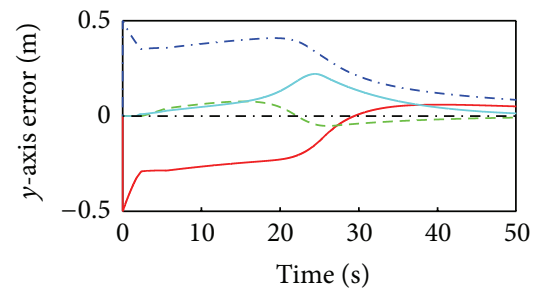

(a)

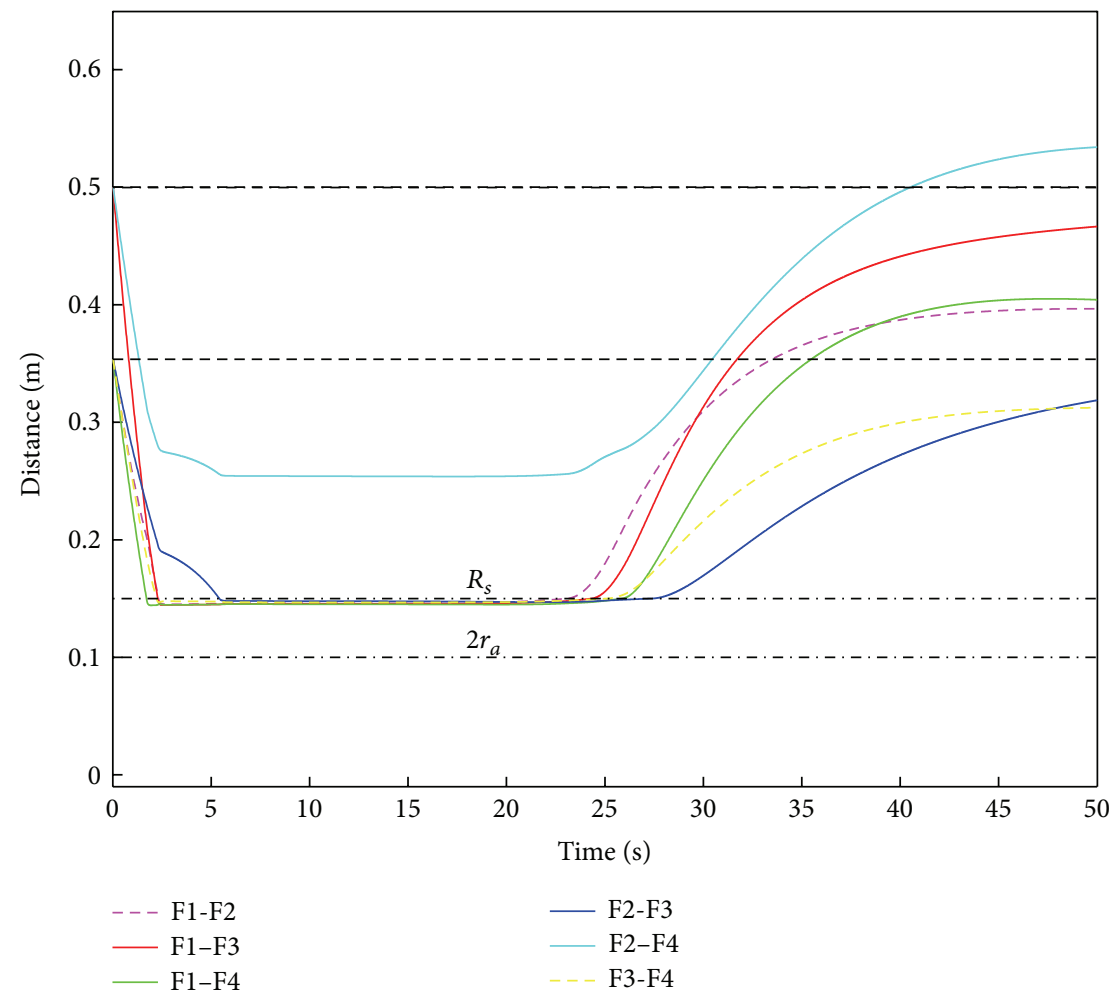

(b)

FIGURE 11: Simulation results of formation and collision avoidance control with static leader (CA + PSC): (a) agents' trajectories and formation errors and (b) the distance between agents. 


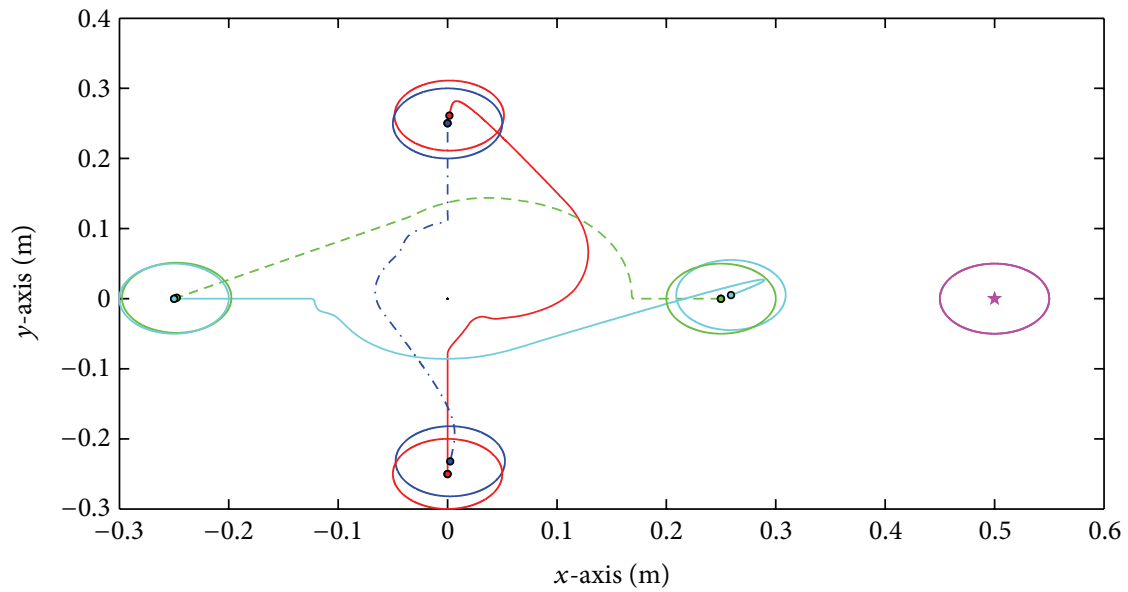

$\star$ Leader $\quad$. . - Follower3

- Follower1 _ Follower4

- - Follower2
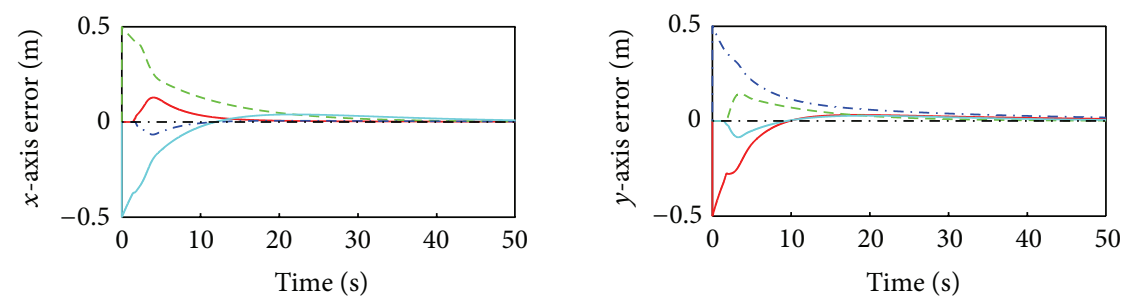

(a)

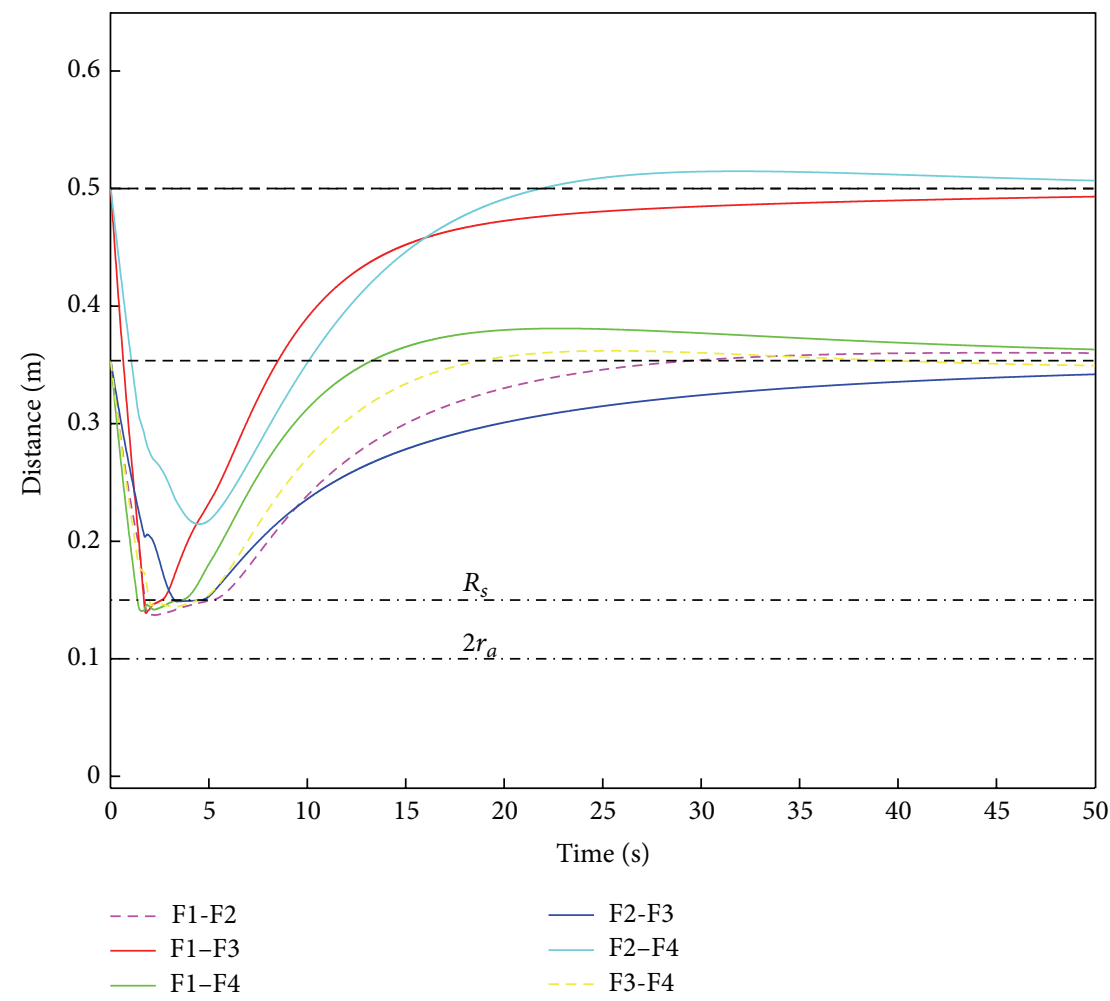

(b)

FIGURE 12: Simulation results of formation and collision-avoidance control with static leader (CA + FSC): (a) agents' trajectories and formation errors and (b) the distance between agents. 

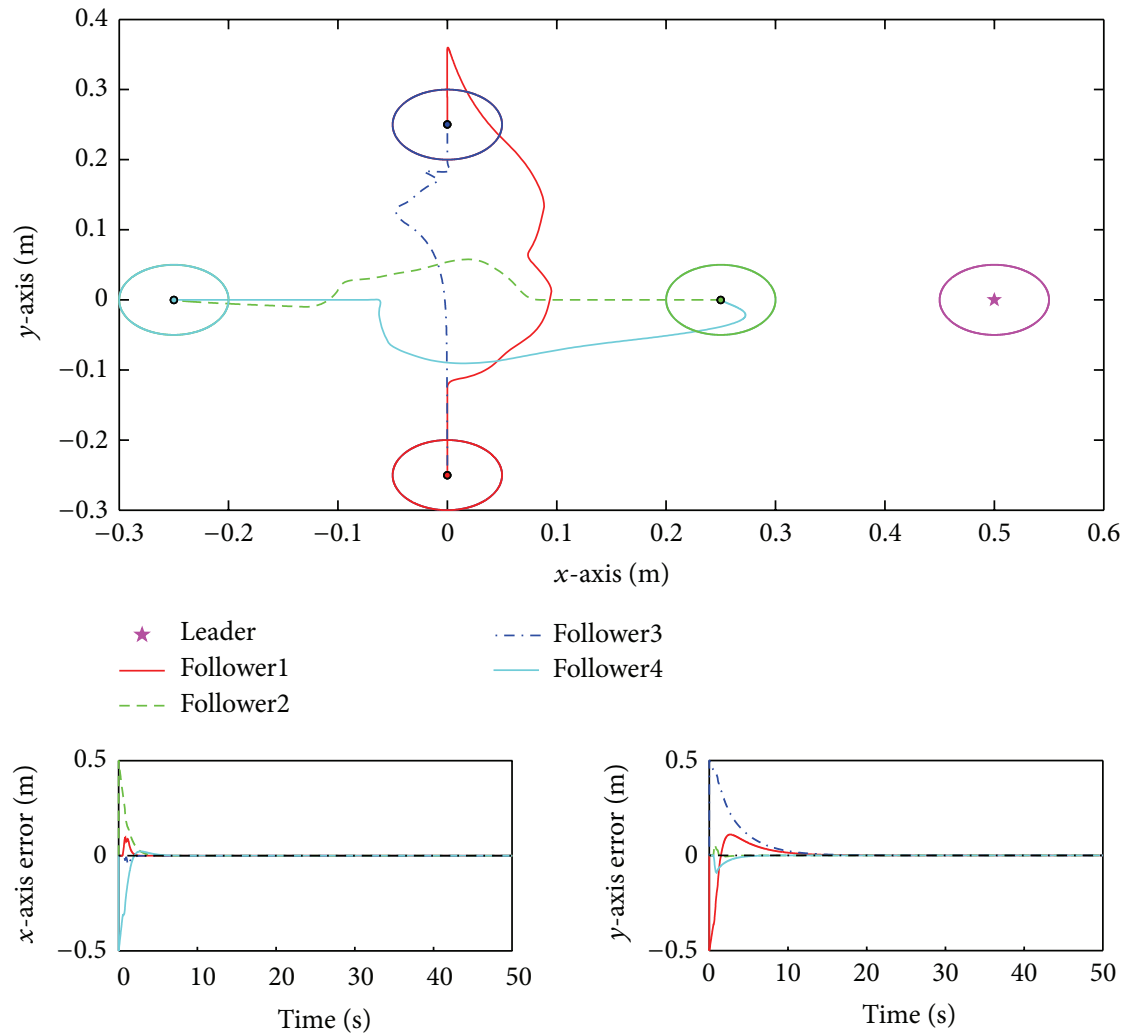

(a)

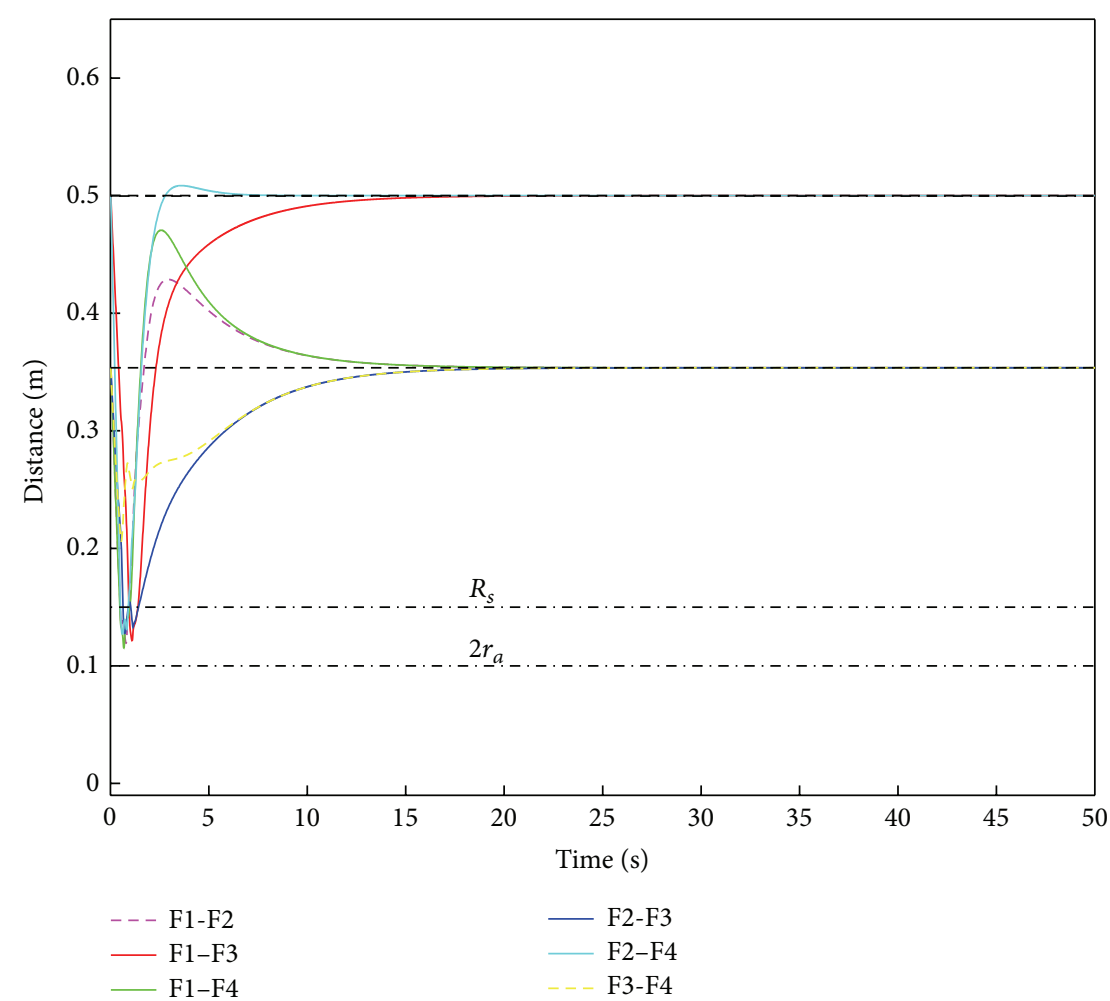

(b)

FIGURE 13: Simulation results of formation and collision-avoidance control with static leader (NFC + FSC): (a) agents' trajectories and formation errors and (b) the distance between agents. 

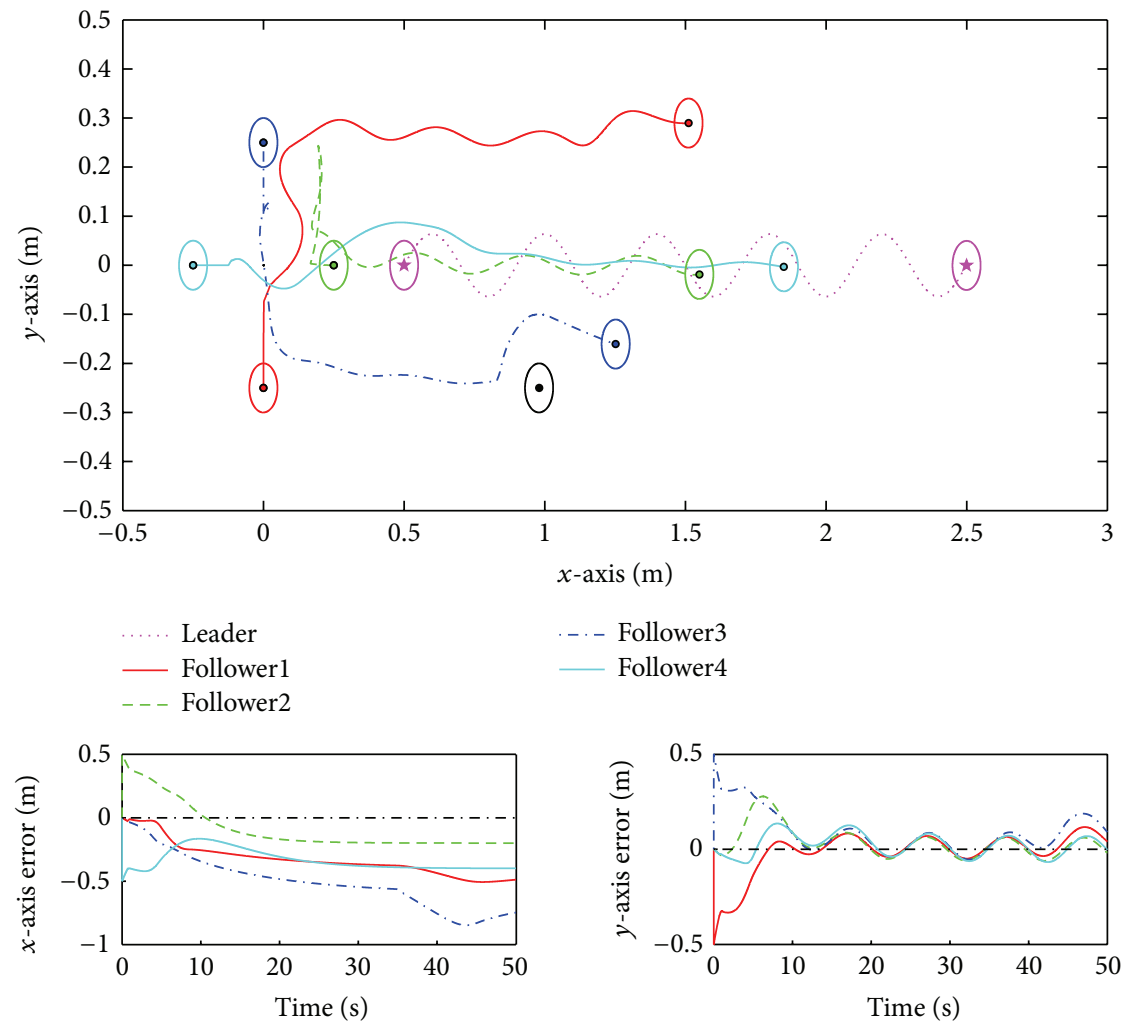

(a)

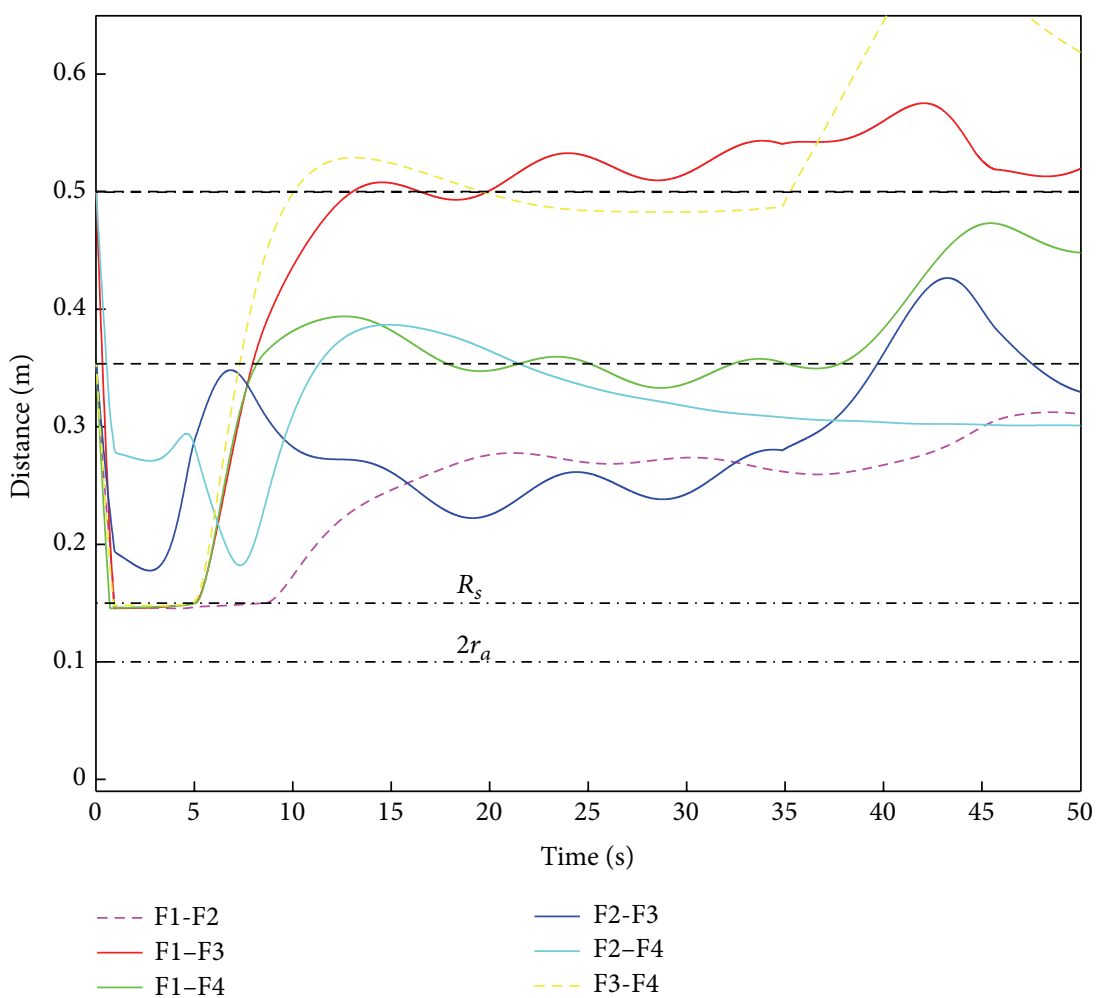

(b)

FIGURE 14: Simulation results of formation and collision-avoidance control with dynamic leader (CA + PSC): (a) agents' trajectories and formation errors and (b) the distance between agents. 

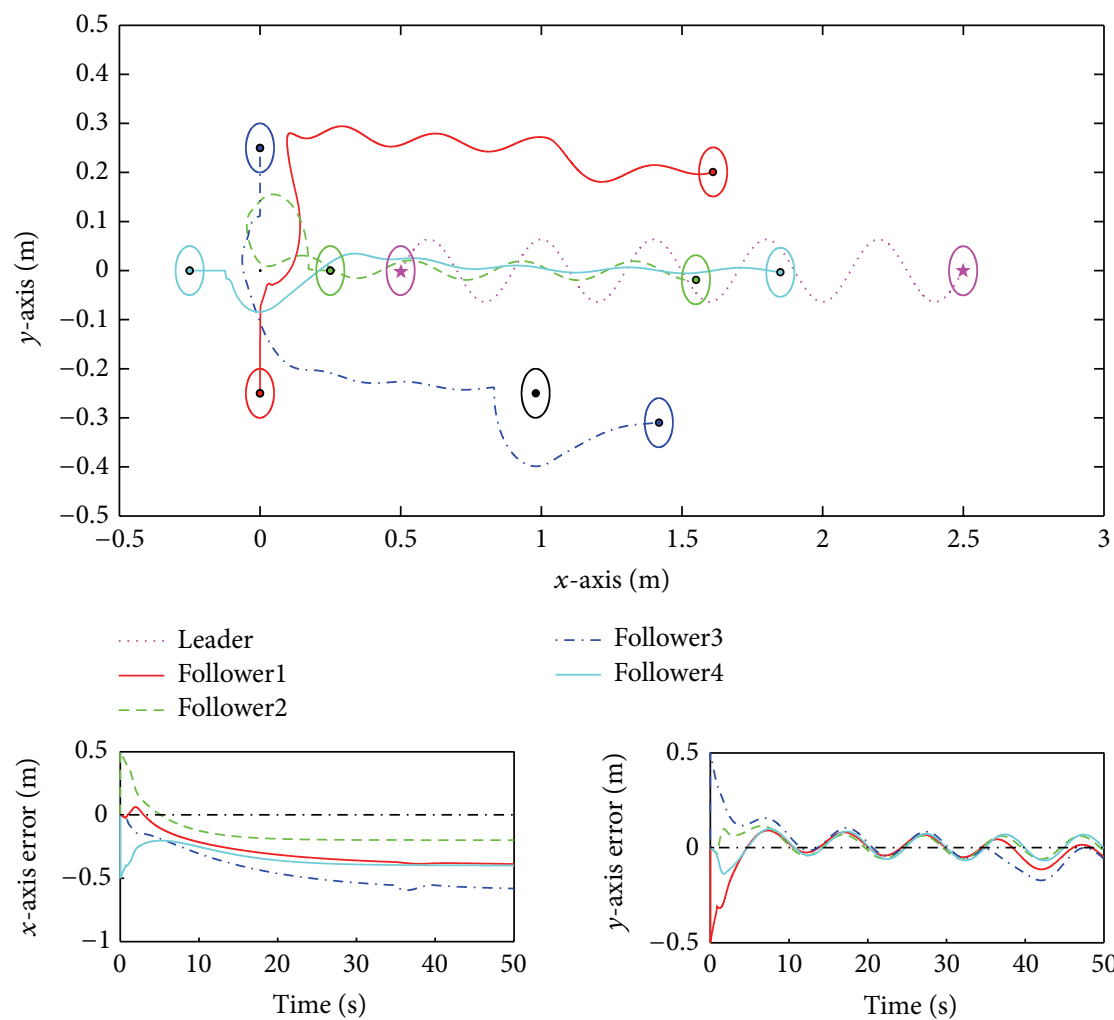

-..- Follower3

- Follower4

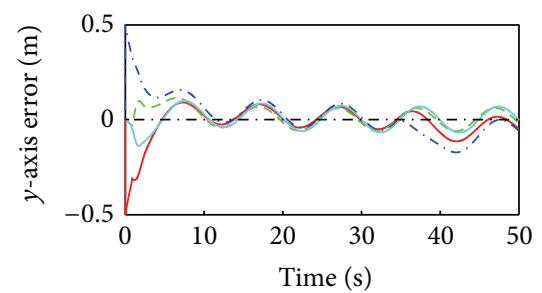

(a)

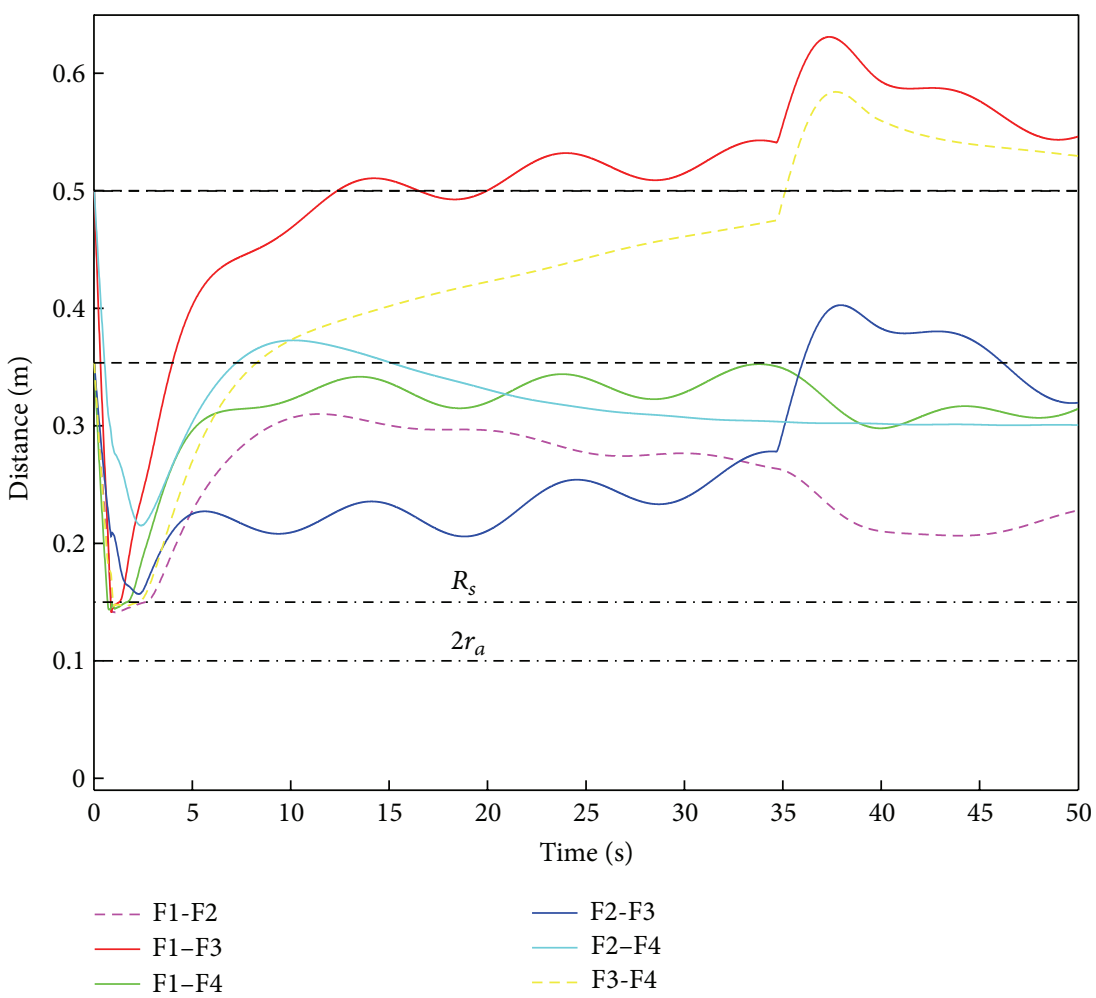

(b)

FIGURE 15: Simulation results of formation and collision-avoidance control with dynamic leader (CA + FSC): (a) agents' trajectories and formation errors and (b) the distance between agents. 

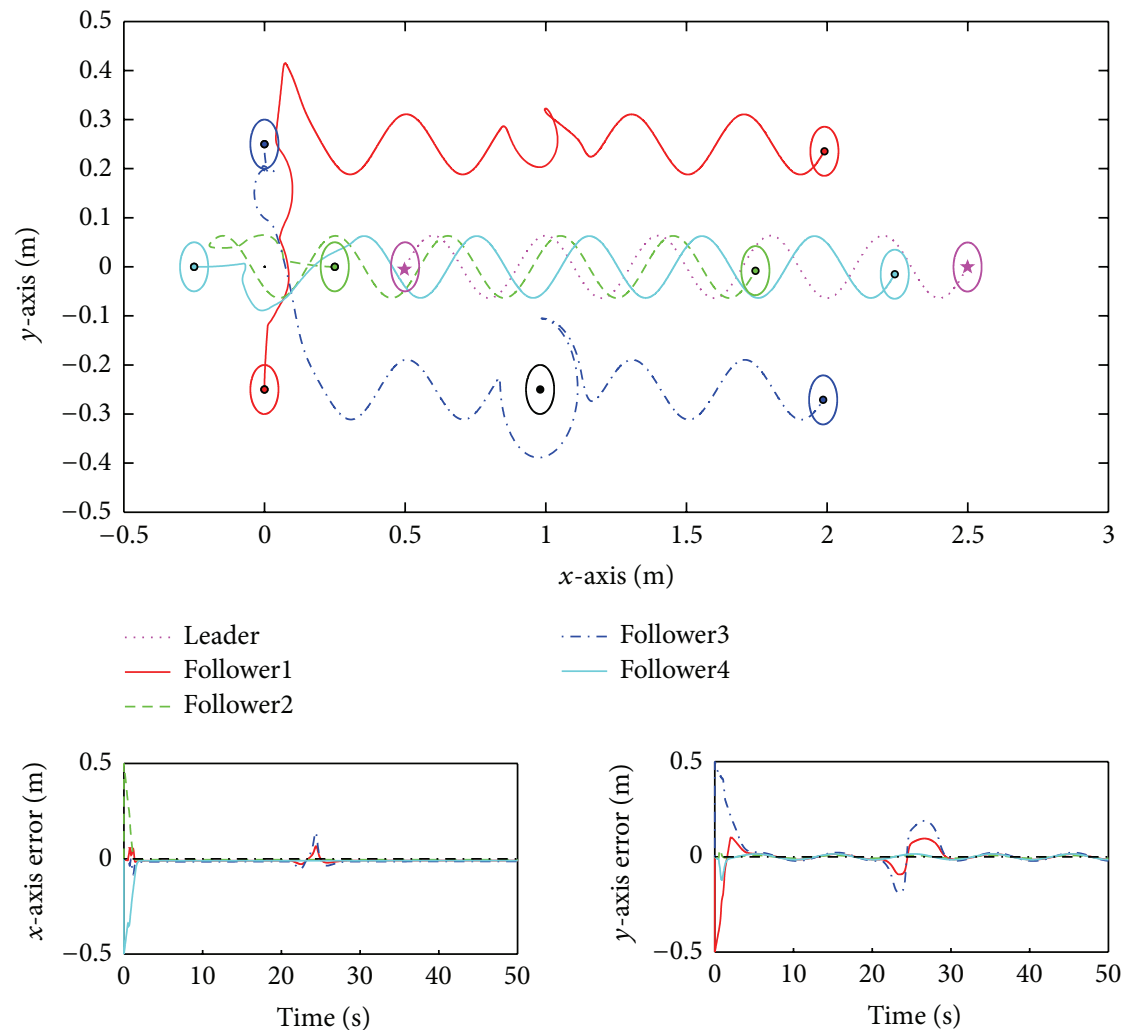

(a)

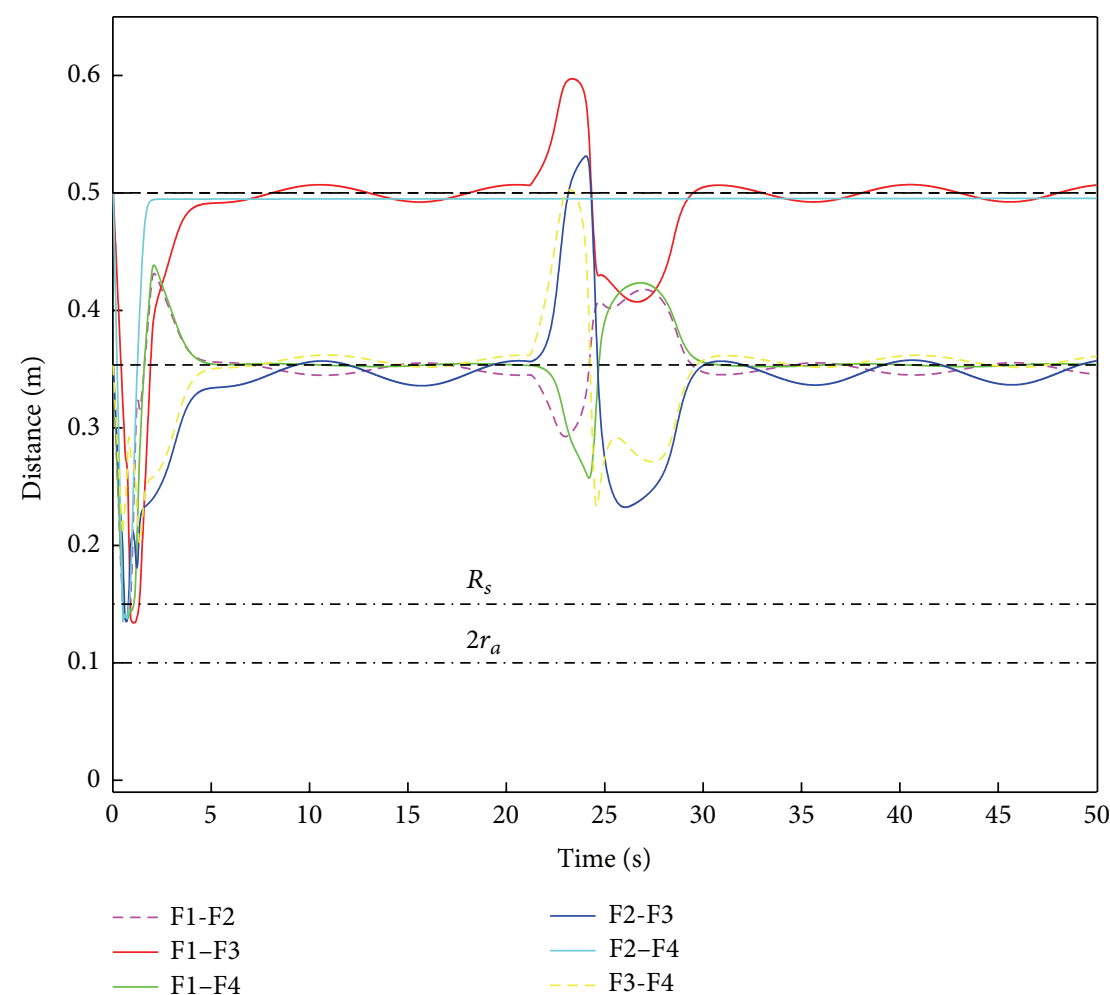

(b)

FIGURE 16: Simulation results of formation and collision-avoidance control with dynamic leader (NFC + FSC): (a) agents' trajectories and formation errors and (b) the distance between agents. 
TABLE 3: Formation errors: formation and separation control with static leader.

\begin{tabular}{lcccc}
\hline & IAE & ISE & ITAE & ITSE \\
\hline CA + PSC & 5887.77 & 1580.74 & 114492.31 & 23911.31 \\
CA + FSC & 1746.77 & 304.78 & 20183.14 & 1249.37 \\
NFC + FSC & 382.47 & 90.12 & 967.88 & 103.77 \\
\hline
\end{tabular}

originally chosen as $c_{1}=3, c_{2}=2, C_{x i}(0)=C_{y i}(0)=\theta_{x i}(0)=$ $\theta_{y i}(0)=\left[\begin{array}{llllll}-1 & -0.67 & -0.33 & 0.33 & 0.67 & 1\end{array}\right]^{T}, i=1,2,3,4$, $\sigma_{x i}(0)=\sigma_{y i}(0)=\left[\begin{array}{llllll}0.5 & 0.5 & 0.5 & 0.5 & 0.5 & 0.5\end{array}\right]^{T}, \eta_{1}=0.022$, $\eta_{2}=0.01$, and $\eta_{3}=0.012$. Three unique distributed strategies, consensus algorithm with potential-based separation control (CA + PSC), consensus algorithm with fuzzy separation control (CA + FSC), and neurofuzzy formation control with fuzzy separation control (NFC + FSC), are considered. Accordingly, simulation results are shown in Figures 11, 12, and 13, including the error trajectories of $x$ - and $y$-axis, and the relative distances between a pair of agents. Since the relative distance between any two agents is greater than $2 r_{a}$, shown in Figures 11(b), 12(b), and 13(b), the desired collision avoidance can be accomplished using these three control strategies. In addition, the formation responses are shown in Figures 11(a), 12(a), and 13(a). It can be seen that there exist significant steady-state errors using CA + PSC. Conversely, the formation pattern can be asymptotically achieved with the use of NFC + FSC. The position errors, $\left|e_{x i}\right|+\left|e_{y i}\right|$, are summarized in Table 3 , where IAE is the integral absolute error, ISE is the integral square error, ITAE is the integral time absolute error, and ITAE stands for the integral time square error. In summary, the proposed NFC + FSC can provide better performance than the counterparts of the other two methods.

5.3. Formation Control and Collision Avoidance with Dynamic Leader. In the following, previous leader-follower system is addressed, where all initial settings are the same except that one fixed agent is added at the position $(0.95,-0.25)(\mathrm{m})$. This fixed agent can be viewed as a standstill obstacle. Besides, a time-varying leader is considered, where the velocity vector is $(0.06,0.08 \sin (0.002 \pi t))(\mathrm{m} / \mathrm{s})$. The formation responses are shown in Figures 14, 15, and 16, where the methods of CA + PSC, CA + FSC, and NFC + FSC are considered. Similar to the previously mentioned illustrations, the moving trajectories and the relative distances between two follower agents are depicted in Figures 14, 15, and 16. It can be seen that even the collision avoidance can be achieved with CA + PSC and CA + FSC; however, these two strategies eventually fail to preserve the desired formation pattern. On the other hand, from Figure 16, collision-free formation can be obtained by using the proposed NFC + FSC control scheme. In particular, it can be seen that the third follower can successfully bypass the fixed agent and keep its way to form a designated pattern. The formation errors of different control strategies are summarized in Table 4. It can be concluded that the proposed neurofuzzy formation and fuzzy separation combined controller can provide better responses
TABLE 4: Formation errors: formation and separation control with dynamic leader.

\begin{tabular}{lcccc}
\hline & IAE & ISE & ITAE & ITSE \\
\hline CA + PSC & 8214.19 & 3156.40 & 223875.11 & 96814.34 \\
CA + FSC & 7477.28 & 2528.48 & 204209.46 & 75349.76 \\
NFC + FSC & 765.87 & 99.88 & 13552.16 & 700.71 \\
\hline
\end{tabular}

than the counterparts of conventional consensus algorithm with potential-based separation control.

Remark 1. From simulation results, it can be seen that the collision-free formation task can be achieved for singleintegrator agents. In particular, the local minimum problem can be overcome using the proposed fuzzy separation control. It is promising that the proposed works can be applied to some practical applications, such as multirobot systems and unmanned vehicle systems.

\section{Conclusion}

This paper presents a neural fuzzy formation controller for multiagent systems. The learning rules for controller parameters can be derived from the use of gradient decent method. In addition, a fuzzy separation control is proposed to achieve collision avoidance such that the problem of local minima can be solved. Simulation results are provided for the cases of static leader and dynamic leader. The collision-free leaderfollower formation can be accomplished by the proposed fuzzy formation and separation control strategy. Performance comparisons indicate that the proposed fuzzy-based control scheme has better formation responses compared to the counterparts of conventional consensus algorithm and potential-based separation control. The current results are mainly limited in single-integrator multiagent systems. It should be challenging and interesting to investigate the collision avoidance problem for high-order agents with nonlinear dynamics. For example, a fuzzy based separation control for kinematic agents will be undertaken in our future work.

\section{Acknowledgments}

This work is partially supported by the Taiwan National Science Council under the Grants NSC 101-2221-E-182-041MY2 and NSC 100-2918-I-182-002. The authors also want to appreciate the High Speed Intelligent Communication Research Center, Chang Gung University, Taiwan, for continuing support in the study of multiagent systems.

\section{References}

[1] Z. G. Hou, L. Cheng, and M. Tan, "Decentralized robust adaptive control for the multiagent system consensus problem using neural networks," IEEE Transactions on Systems, Man, and Cybernetics, Part B, vol. 39, no. 3, pp. 636-647, 2009.

[2] H. Zhang and F. L. Lewis, "Adaptive cooperative tracking control of higher-order nonlinear systems with unknown dynamics," Automatica, vol. 48, no. 7, pp. 1432-1439, 2012. 
[3] M. E. Valcher and P. Misra, "On the controllability and stabilizability of non-homogeneous multi-agent dynamical systems," Systems \& Control Letters, vol. 61, no. 7, pp. 780-787, 2012.

[4] B. Ranjbar-Sahraei, F. Shabaninia, A. Nemati, and S.-D. Stan, "A novel robust decentralized adaptive fuzzy control for swarm formation of multiagent systems," IEEE Transactions on Industrial Electronics, vol. 59, no. 8, pp. 3124-3134, 2012.

[5] R. Cui, B. Ren, and S. S. Ge, "Synchronised tracking control of multi-agent system with high-order dynamics," IET Control Theory \& Applications, vol. 6, no. 5, pp. 603-614, 2012.

[6] X. Luo, D. Liu, X. Guan, and S. Li, "Flocking in target pursuit for multi-agent systems with partial informed agents," IET Control Theory \& Applications, vol. 6, no. 4, pp. 560-569, 2012.

[7] D. J. Pack, P. DeLima, G. J. Toussaint, and G. York, "Cooperative control of UAVs for localization of intermittently emitting mobile targets," IEEE Transactions on Systems, Man, and Cybernetics, Part B, vol. 16, no. 1, pp. 19-33, 2009.

[8] T. Keviczky, F. Borrelli, K. Fregene, D. Godbole, and G. J. Balas, "Decentralized receding horizon control and coordination of autonomous vehicle formations," IEEE Transactions on Control Systems Technology, vol. 16, no. 1, pp. 19-33, 2008.

[9] K. S. Hwang, S. W. Tan, M. C. Hsiao, and C. S. Wu, "Cooperative multiagent congestion control for high-speed networks," IEEE Transactions on Systems, Man, and Cybernetics, Part B, vol. 35, no. 2, pp. 255-268, 2005.

[10] L. Shi, A. Capponi, K. H. Johansson, and R. M. Murray, "Resource optimisation in a wireless sensor network with guaranteed estimator performance," IET Control Theory and Applications, vol. 4, no. 5, pp. 710-723, 2010.

[11] B. Fidan, C. Yu, and B. D. O. Anderson, "Acquiring and maintaining persistence of autonomous multi-vehicle formations," IET Control Theory and Applications, vol. 1, no. 2, pp. 452-460, 2007.

[12] R. Olfati-Saber and R. M. Murray, "Consensus problems in networks of agents with switching topology and time-delays," IEEE Transactions on Automatic Control, vol. 49, no. 9, pp. 1520 1533, 2004.

[13] H. Jiang, J. Yu, and C. Zhou, "Consensus of multi-agent linear dynamic systems via impulsive control protocols," International Journal of Systems Science, vol. 42, no. 6, pp. 967-976, 2011.

[14] Y.-H. Chang, C.-W. Chang, C.-L. Chen, and C.-W. Tao, "Fuzzy sliding-mode formation control for multi-robot systems: design and implementation," IEEE Transactions on Systems, Man and Cybernetics, Part B, vol. 42, no. 2, pp. 444-457, 2012.

[15] N. Cai, J.-X. Xi, Y.-S. Zhong, and H.-Y. Ma, "Controllability improvement for linear time-invariant dynamic multi-agent systems," International Journal of Innovative Computing, Information and Control, vol. 8, no. 5, pp. 3315-3328, 2012.

[16] W. Ni and D. Cheng, "Leader-following consensus of multiagent systems under fixed and switching topologies," Systems and Control Letters, vol. 59, no. 3-4, pp. 209-217, 2010.

[17] J. Qin, H. Gao, and W. X. Zheng, "Second-order consensus for multi-agent systems with switching topology and communication delay," Systems and Control Letters, vol. 60, no. 6, pp. 390397, 2011.

[18] F. Jiang, L. Wang, and G. Xie, "Consensus of high-order dynamic multi-agent systems with switching topology and time-varying delays," Journal of Control Theory and Applications, vol. 8, no. 1, pp. 52-60, 2010.

[19] Y. Zheng, Y. Zhu, and L. Wang, "Consensus of heterogeneous multi-agent systems," IET Control Theory \& Applications, vol. 5, no. 16, pp. 1881-1888, 2011.
[20] X. Su, P. Shi, L. Wu, and Y.-D. Song, "A novel control design on discrete-time Takagi-Sugeno fuzzy systems with time-varying delays," IEEE Transactions on Fuzzy Systems, 2012.

[21] X. Su, P. Shi, L. Wu, and Y.-D. Song, "A novel approach to filter design for T-S fuzzy discrete-time systems with time-varying delay," IEEE Transactions on Fuzzy Systems, vol. 20, no. 6, pp. 1114-1129, 2012.

[22] X. Su, P. Shi, L. Wu, and S. K. Nguang, "Induced $\ell_{2}$ filtering of fuzzy stochastic systems with time-varying delays," IEEE Transactions on Systems, Man and Cybernetics, Part B, 2012.

[23] L. Wu, X. Su, P. Shi, and J. Qiu, "Model approximation for discrete-time state-delay systems in the TS fuzzy framework," IEEE Transactions on Fuzzy Systems, vol. 19, no. 2, pp. 366-378, 2011.

[24] $\mathrm{L}$. Wu and W. X. Zheng, " $L_{2}-L_{\infty}$ control of nonlinear fuzzy itô stochastic delay systems via dynamic output feedback," IEEE Transactions on Systems, Man, and Cybernetics, Part B, vol. 39, no. 5, pp. 1308-1315, 2009.

[25] L. Wu, X. Su, P. Shi, and J. Qiu, "A new approach to stability analysis and stabilization of discrete-time T-S fuzzy time-varying delay systems," IEEE Transactions on Systems, Man, and Cybernetics, Part B, vol. 41, no. 1, pp. 273-286, 2011.

[26] R. J. Wai and J. D. Lee, "Robust levitation control for linear Maglev rail system using fuzzy neural network," IEEE Transactions on Control Systems Technology, vol. 17, no. 1, pp. 4-14, 2009.

[27] X. Yuan, Y. Wang, L. Wu, X. Zhang, and W. Sun, "Neural network based self-learning control strategy for electronic throttle valve," IEEE Transactions on Vehicular Technology, vol. 59, no. 8, pp. 3757-3765, 2010.

[28] F. J. Lin and P. H. Shen, "Adaptive fuzzy-neural-network control for a DSP-based permanent magnet linear synchronous motor servo drive," IEEE Transactions on Fuzzy Systems, vol. 14, no. 4, pp. 481-495, 2006.

[29] M. Singh and A. Chandra, "Application of adaptive networkbased fuzzy inference system for sensorless control of PMSGbased wind turbine with nonlinear-load-compensation capabilities," IEEE Transactions on Power Electronics, vol. 26, no. 1, pp. 165-175, 2011.

[30] S. P. Moustakidis, G. A. Rovithakis, and J. B. Theocharis, "An adaptive neuro-fuzzy tracking control for multi-input nonlinear dynamic systems," Automatica, vol. 44, no. 5, pp. 1418-1425, 2008.

[31] S. H. Park and S. I. Han, "Robust-tracking control for robot manipulator with deadzone and friction using backstepping and RFNN controller," IET Control Theory \& Applications, vol. 5, no. 12, pp. 1397-1417, 2011.

[32] C.-S. Chen, "Robust self-organizing neural-fuzzy control with uncertainty observer for MIMO nonlinear systems," IEEE Transactions on Fuzzy Systems, vol. 19, no. 4, pp. 694-706, 2011.

[33] M. Khezri and M. Jahed, "A neuro-fuzzy inference system for sEMG-based identification of hand motion commands," IEEE Transactions on Industrial Electronics, vol. 58, no. 5, pp. 19521960, 2011.

[34] C. Chen, B. Zhang, and G. Vachtsevanos, "Prediction of machine health condition using neuro-fuzzy and Bayesian algorithm," IEEE Transactions on Instrumentation and Measurement, vol. 61, no. 2, pp. 297-306, 2012.

[35] W.-Y. Wang, M.-C. Chen, and S.-F. Su, "Hierarchical T-S fuzzyneural control of anti-lock braking system and active suspension in a vehicle," Automatica, vol. 48, no. 8, pp. 1698-1706, 2012.

[36] T. Niknam, H. D. Mojarrad, and M. Nayeripour, "A new hybrid fuzzy adaptive particle swarm optimization for nonconvex economic dispatch," International Journal of Innovative 
Computing, Information and Control, vol. 7, no. 1, pp. 189-202, 2011.

[37] D. M. Stipanović, P. F. Hokayem, M. W. Spong, and D. D. Šiljak, "Cooperative avoidance control for multiagent systems," Journal of Dynamic Systems, Measurement and Control, Transactions of the ASME, vol. 129, no. 5, pp. 699-707, 2007.

[38] D. Gu and H. Hu, "Using fuzzy logic to design separation function in flocking algorithms," IEEE Transactions on Fuzzy Systems, vol. 16, no. 4, pp. 826-838, 2008.

[39] D. Gu and Z. Wang, "Leader-follower flocking: algorithms and experiments," IEEE Transactions on Control Systems Technology, vol. 17, no. 5, pp. 1211-1219, 2009.

[40] P. F. Hokayem, D. M. Stipanović, and M. W. Spong, "Coordination and collision avoidance for Lagrangian systems with disturbances," Applied Mathematics and Computation, vol. 217, no. 3, pp. 1085-1094, 2010.

[41] S. S. Ge and Y. J. Cui, "Dynamic motion planning for mobile robots using potential field method," Autonomous Robots, vol. 13, no. 3, pp. 207-222, 2002.

[42] Z. Wang and D. Gu, "Cooperative target tracking control of multiple robots," IEEE Transactions on Industrial Electronics, vol. 59, no. 8, pp. 3232-3240, 2012.

[43] M. Mesbahi and M. Egerstedt, Graph Theoretic Methods in Multiagent Networks, Princeton University Press, Princeton, NJ, USA, 2010.

[44] L. Khan, S. Anjum, and R. Badar, "Standard fuzzy model identification using gradient methods," World Applied Sciences Journal, vol. 8, no. 1, pp. 1-9, 2010.

[45] F. J. Lin, R. J. Wai, and C. C. Lee, "Fuzzy neural network position controller for ultrasonic motor drive using push-pull DC-DC converter," IEE Proceedings: Control Theory and Applications, vol. 146, no. 1, pp. 99-107, 1999.

[46] W. Ren and R. Beard, Distributed Consensus in Multi-Vehicle Cooperative Control: Theory and Applications, Springer, 2007. 


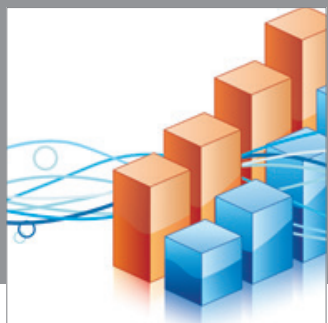

Advances in

Operations Research

mansans

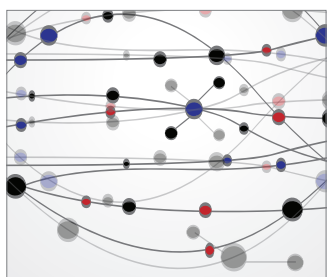

The Scientific World Journal
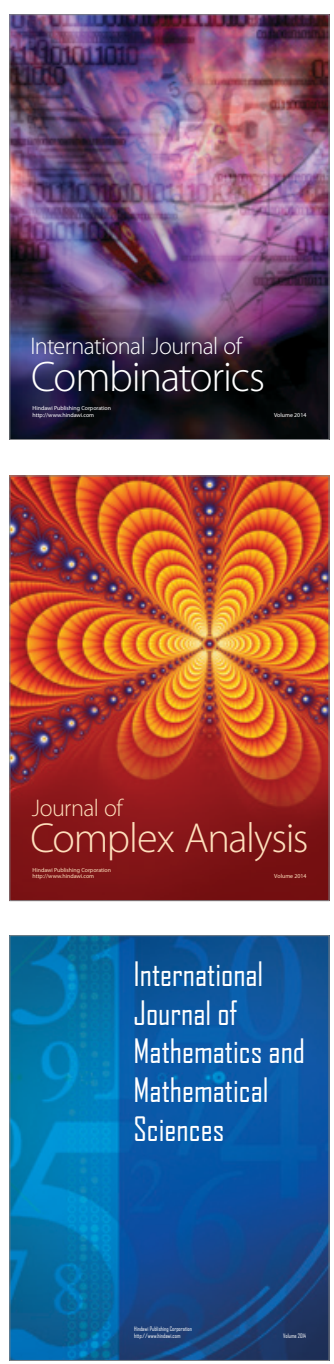
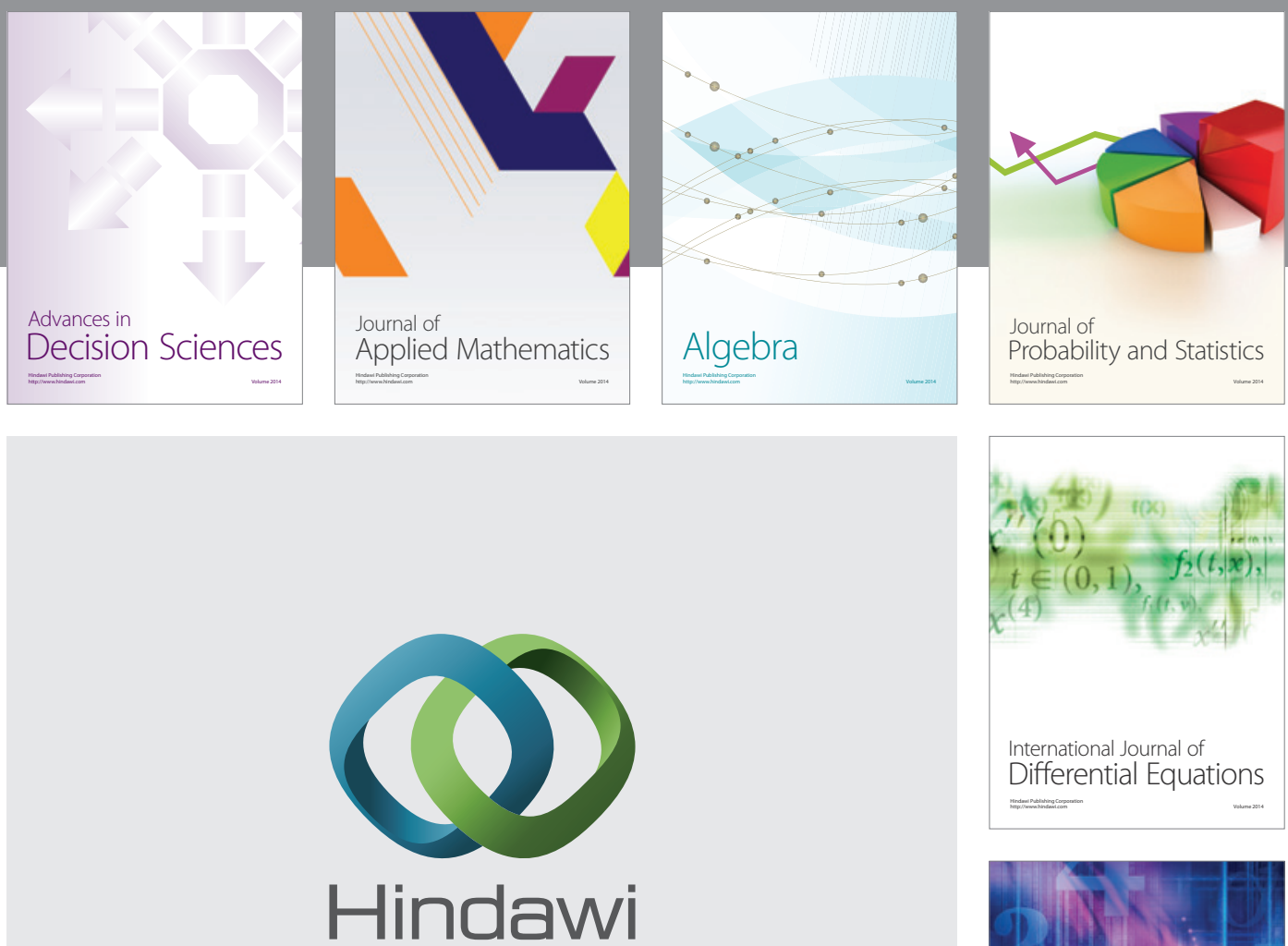

Submit your manuscripts at http://www.hindawi.com
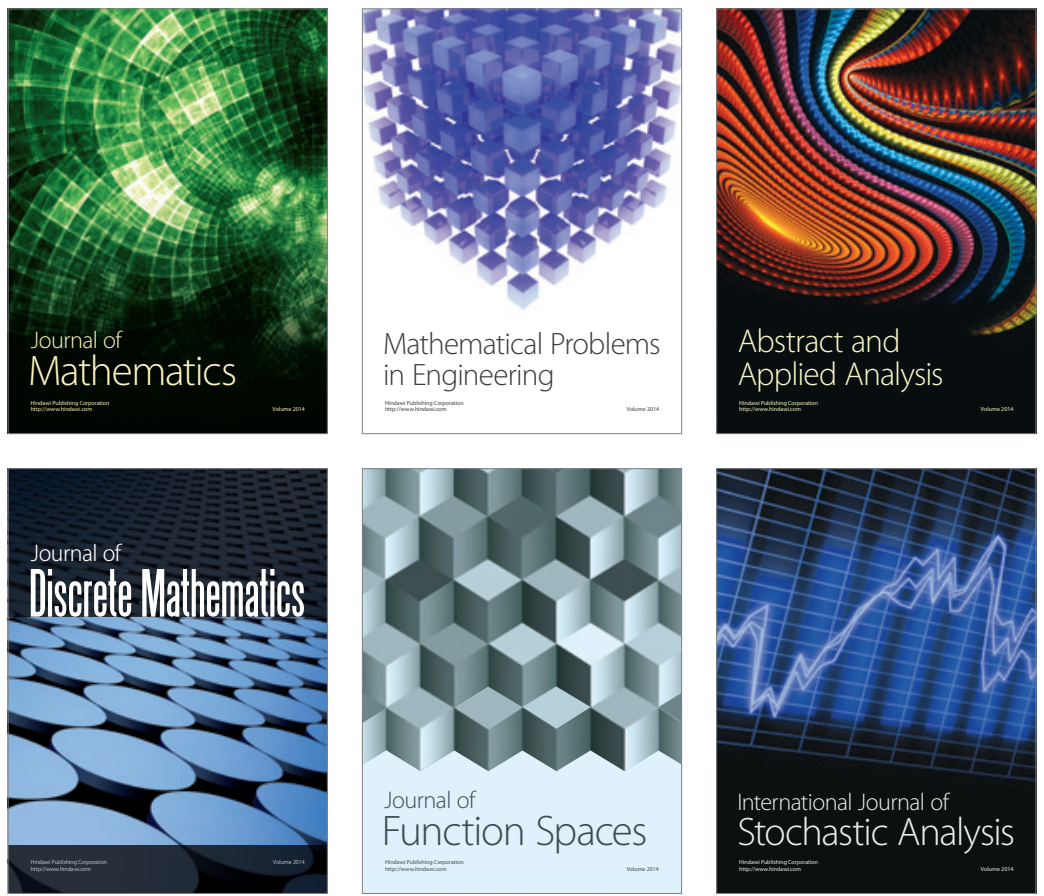

Journal of

Function Spaces

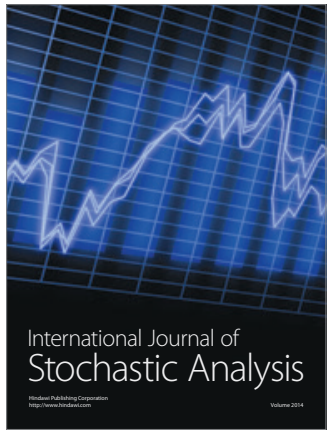

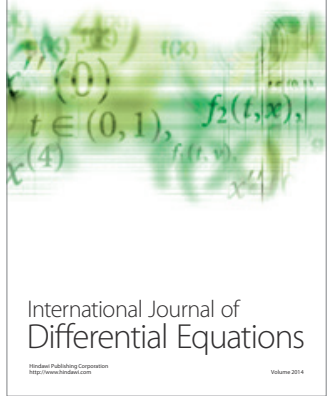
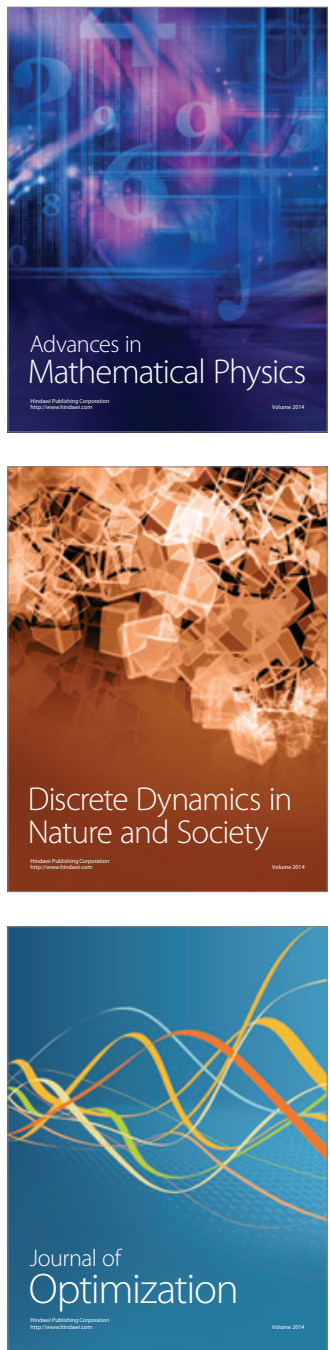